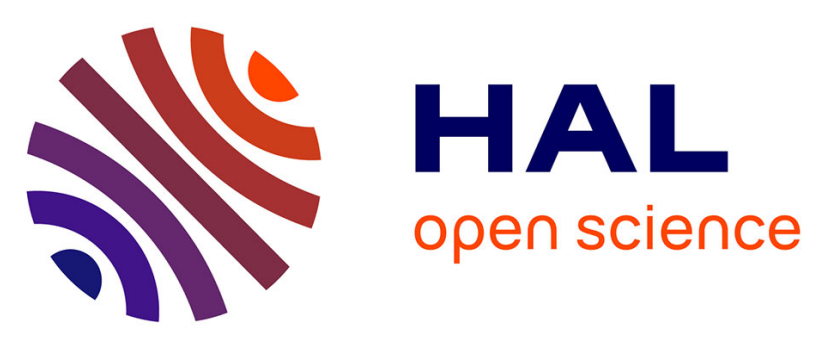

\title{
Modeling Human Neural Functionality In Vitro: Three-Dimensional Culture for Dopaminergic Differentiation
}

\author{
Daniel Simão, Catarina Pinto, Stefania Piersanti, Anne Weston, Christopher \\ Peddie, André E.P. Bastos, Valerio Licursi, Sigrid C. Schwarz, Lucy Collinson, \\ Sara Salinas, et al.
}

\section{To cite this version:}

Daniel Simão, Catarina Pinto, Stefania Piersanti, Anne Weston, Christopher Peddie, et al.. Modeling Human Neural Functionality In Vitro: Three-Dimensional Culture for Dopaminergic Differentiation. Tissue Engineering: Parts A, B, and C, 2015, 21 (3-4), pp.654-668. 10.1089/ten.TEA.2014.0079 . hal-01990919

\section{HAL Id: hal-01990919 \\ https://hal.umontpellier.fr/hal-01990919}

Submitted on 24 Jan 2019

HAL is a multi-disciplinary open access archive for the deposit and dissemination of scientific research documents, whether they are published or not. The documents may come from teaching and research institutions in France or abroad, or from public or private research centers.
L'archive ouverte pluridisciplinaire HAL, est destinée au dépôt et à la diffusion de documents scientifiques de niveau recherche, publiés ou non, émanant des établissements d'enseignement et de recherche français ou étrangers, des laboratoires publics ou privés. 


\title{
Modeling Human Neural Functionality In Vitro: Three-Dimensional Culture for Dopaminergic Differentiation
}

\author{
Daniel Simão, MSc, ${ }^{1,2,{ }^{*}}$ Catarina Pinto, MSc, ${ }^{1,2, *}$ Stefania Piersanti, PhD, ${ }^{3}$ Anne Weston, $\mathrm{PhD},{ }^{4}$ \\ Christopher J. Peddie, PhD, ${ }^{4}$ André E.P. Bastos, MSc, ${ }^{5,6}$ Valerio Licursi, PhD, ${ }^{3}$ Sigrid C. Schwarz, PhD, ${ }^{7}$ \\ Lucy M. Collinson, $\mathrm{PhD},{ }^{4}$ Sara Salinas, $\mathrm{PhD},{ }^{8,9}$ Margarida Serra, $\mathrm{PhD},{ }^{1,2}$ Ana P. Teixeira, $\mathrm{PhD},{ }^{1,2}$ \\ Isabella Saggio, PhD, ${ }^{3,10,11}$ Pedro A. Lima, PhD, ${ }^{5}$ Eric J. Kremer, PhD, ${ }^{8,9}$ Giampietro Schiavo, PhD, ${ }^{4,12}$ \\ Catarina Brito, $\mathrm{PhD}^{1,2}$ and Paula M. Alves, $\mathrm{PhD}^{1,2}$
}

\begin{abstract}
Advances in mechanistic knowledge of human neurological disorders have been hindered by the lack of adequate human in vitro models. Three-dimensional (3D) cellular models displaying higher biological relevance are gaining momentum; however, their lack of robustness and scarcity of analytical tools adapted to three dimensions hampers their widespread implementation. Herein we show that human midbrain-derived neural progenitor cells, cultured as 3D neurospheres in stirred culture systems, reproducibly differentiate into complex tissue-like structures containing functional dopaminergic neurons, as well as astrocytes and oligodendrocytes. Moreover, an extensive toolbox of analytical methodologies has been adapted to 3D neural cell models, allowing molecular and phenotypic profiling and interrogation. The generated neurons underwent synaptogenesis and elicit spontaneous $\mathrm{Ca}^{2+}$ transients. Synaptic vesicle trafficking and release of dopamine in response to depolarizing stimuli was also observed. Under whole-cell current-and-voltage clamp, recordings showed polarized neurons $\left(V_{\mathrm{m}}=-70 \mathrm{mV}\right)$ and voltage-dependent potassium currents, which included A-type-like currents. Glutamate-induced currents sensitive to $\alpha$-amino-3-hydroxy-5-methyl-4-isoxazolepropionic acid and $\mathrm{N}$-methyl-D-aspartate antagonists revealed the existence of functional glutamate receptors. Molecular and phenotypic profiling showed recapitulation of midbrain patterning events, and remodeling toward increased similarity to human brain features, such as extracellular matrix composition and metabolic signature. We have developed a robust and reproducible human 3D neural cell model, which may be extended to patient-derived induced pluripotent stem cells, broadening the applicability of this model.
\end{abstract}

\section{Introduction}

$\mathbf{T}$ HE DEMAND FOR ROBUST and predictable human in vitro models that can bridge the gap between human clinical studies and animal models is steadily increasing. Advances in our understanding of human diseases as well as drug development have been hindered by the lack of reliable model systems. ${ }^{1,2}$ The study of central nervous system
(CNS) disorders, such as Parkinson's disease (PD), would particularly benefit from the development of more accurate cellular models, since only about $8 \%$ of new drugs that enter clinical trials are approved and reach the market. ${ }^{3}$ In the case of PD, the available chemical or genetically induced animal models poorly mimic most pathological features, due to fundamental biochemical, metabolic, and genetic differences between species. ${ }^{4}$ Important advances have been

\footnotetext{
${ }^{1}$ iBET_-Instituto de Biologia Experimental e Tecnológica, Oeiras, Portugal.

${ }^{2}$ Instituto de Tecnologia Química e Biológica, Universidade Nova de Lisboa, Oeiras, Portugal.

${ }^{3}$ Dipartimento di Biologia e Biotecnologie "Charles Darwin," Università di Roma La Sapienza, Rome, Italy.

${ }_{5}^{4}$ Lincoln's Inn Fields Laboratories, Cancer Research UK London Research Institute, London, United Kingdom.

${ }^{5}$ NOVA Medical School, Faculdade de Ciências Médicas da Universidade Nova de Lisboa, Lisboa, Portugal.

${ }^{6}$ Departamento de Química e Bioquímica, Faculdade de Ciências da Universidade de Lisboa, Lisboa, Portugal.

${ }^{7}$ Department of Neurology, University of Leipzig, Leipzig, Germany.

${ }^{8}$ Institut de Génétique Moléculaire de Montpellier, CNRS UMR 5535, Montpellier, France.

${ }^{9}$ Université Montpellier I and II, Montpellier, France.

${ }^{10}$ Istituto Pasteur Fondazione Cenci Bolognetti, Università di Roma La Sapienza, Rome, Italy.

${ }^{11}$ Istituto di Biologia e Patologia Molecolari del CNR, Università di Roma La Sapienza, Rome, Italy.

${ }^{12}$ Sobell Department of Motor Neuroscience and Movement Disorders, Institute of Neurology, University College London, London, United Kingdom.

*These two authors contributed equally to this work.
} 
made toward the development of human cellular models using immortalized cell lines, embryonic or adult neural stem cells and, more recently, induced pluripotent stem cells (iPSCs). ${ }^{4}$

Cell fate is determined by processes that integrate a wide range of external cues, such as nutritional status, growth factors, mechanical cues, cell-cell, and cell-extracellular matrix (ECM) interactions. ${ }^{2,5}$ Thus, when aiming at mimicking the main features of tissues, it is imperative to establish three-dimensional (3D) cellular networks, which play critical roles in cell fate, tissue specificity, and homeostasis. ${ }^{6}$ Therefore, in vitro cellular models with a higher spatial degree of complexity are necessary. With the growing set of platforms amenable to high-throughput screening (HTS), as well as the increasing power of methodologies that allow more comprehensive readouts, human $3 \mathrm{D}$ ex vivo models can contribute to generate accurate and predictive cell-based drug and toxicity screenings. Several cellular systems have been established, namely, organotypic cultures, which fail to maintain long-term viability, and 3D in vitro cell cultures, either within matrices, highly dependent on time-consuming scaffold engineering and preparation, ${ }^{7}$ or as free-floating aggregates. $^{6}$

By taking advantage of the potential of many cell types to self-organize into 3D structures, with secretion of ECM, ${ }^{5,8}$ one may culture isolated cells as 3D spheroids, ${ }^{5,6}$ which in case of neural cultures are referred to as neurospheres and have been reported to mimic basic processes of brain development. ${ }^{9}$ Different methods for aggregation have been explored, including spontaneous aggregation under static conditions and in rotating wall vessels or induced aggregation in stirred culture systems. ${ }^{5}$ As shown by our group, human midbrain-derived neural progenitor cells (hmNPCs) from fetal origin can be successfully cultured in low oxygen and serum-free medium as neurospheres in stirred culture systems and differentiated toward the dopaminergic lineage. ${ }^{10}$

In this work, we further explored this dynamic culture system in order to attain more efficient dopaminergic differentiation and neuronal maturation, along with a comprehensive set of characterization methods adapted for a 3D setting. By extending culture time in the presence of cAMP, we were able to establish a reliable $3 \mathrm{D}$ differentiation process in which dopaminergic and synaptic markers were upregulated, recapitulating key events of midbrain development. Moreover, the expression of synaptic markers and their assembly in synaptic-like microvesicles resulted in increased neuronal functionality, as suggested by the ability to spontaneously elicit $\mathrm{Ca}^{2+}$-firing and respond to depolarizing stimuli, analyzed by $3 \mathrm{D}$ live imaging. The functionality of the generated neurons was further confirmed by the appearance of hyperpolarized resting potentials, voltageactivated currents, and glutamate-evoked currents. Therefore, this 3D human neural model is biologically relevant, and it can be exploited for different applications ranging from mechanistic studies on disease pathogenesis to drug screening.

\section{Materials and Methods}

\section{Two-dimensional cell expansion}

hmNPCs derived from aborted fetal brain tissue 1214 weeks postfertilization ${ }^{10,11}$ were kindly provided by
Dr. Johannes Schwarz (Technical University of Munich). Tissue was obtained with mother's consent and in accordance with the ethics committee of the University of Leipzig and the German state and federal laws. Expansion of hmNPCs was performed on poly-L-ornithine-fibronectin (PLOF)-coated surfaces and serum-free medium, as described previously. ${ }^{10,11}$ Expansion medium (EM) was composed of Dulbecco's modified Eagle medium and Ham's F12 Nutrient Mix (both from Invitrogen) in a 1:1 ratio, 2\% B27 supplement (Invitrogen), $20 \mathrm{ng} / \mathrm{mL}$ recombinant human (rhu)-fibroblast growth factor (FGF) 2 and rhu-epidermal growth factor (EGF) (both from PrepoTech), $1 \mu \mathrm{g} / \mathrm{mL}$ tocopherol (Fluka), $1 \mu \mathrm{g} / \mathrm{mL}$ tocopherol acetate (Sigma), and $10 \mu \mathrm{g} / \mathrm{mL}$ gentamycin (Invitrogen). Cells were maintained in a multigas cell incubator (Sanyo) at $37^{\circ} \mathrm{C}$, in a humidified atmosphere of $5 \% \mathrm{CO}_{2}$ and $3 \% \mathrm{O}_{2}$ in air. $\mathrm{A}$ $100 \%$ media exchange was performed every $3-4$ days. Splitting was typically performed every 14 days, at $90-100 \%$ confluence, dislodging cells through incubation with Accutase (Sigma). The cell suspension was used to inoculate PLOF-coated $\mathrm{T}$-flasks, at a cell density of $3 \times 10^{4} \mathrm{cell} / \mathrm{cm}^{2}$.

\section{D neurosphere differentiation}

hmNPCs were cultured in dynamic culture systems, using shake flasks (Corning) under constant orbital shaking (stirring rate $=100 \mathrm{rpm}$ ) at $37^{\circ} \mathrm{C}$ in a multigas cell incubator (Sanyo), with a humidified atmosphere of $5 \% \mathrm{CO}_{2}$ and $3 \%$ $\mathrm{O}_{2}$ in air. Typically, flasks were inoculated at $2 \times 10^{5}$ cells/ $\mathrm{mL}$ (single-cell suspension) in aggregation medium (AM; EM with lower mitogen concentration $5 \mathrm{ng} / \mathrm{mL}$ of FGF2 and EGF) and maintained in AM for 7 days with a $50 \%$ medium exchange performed at days 3-4. At day 7 of aggregation (7Diff), differentiation of neurospheres was induced by exchanging AM to differentiation medium (DM; neurobasal medium [Invitrogen] supplemented with $2 \%$ of B27, $2 \mathrm{mM}$ of Glutamax [Invitrogen], $100 \mu \mathrm{M}$ dibutyryl c-AMP [SigmaAldrich], $10 \mu \mathrm{M}$ forskolin [Sigma-Aldrich], $100 \mu \mathrm{M}$ fusaric acid [Sigma-Aldrich], and $10 \mu \mathrm{g} / \mathrm{mL}$ gentamycin [Invitro-

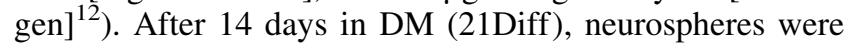
further cultured in maturation medium (MM; same composition of DM, except for removal of forskolin and fusaric acid) for 18 days (18Mat). A 75\% medium exchange was performed every 2-3 days.

\section{Viability assay}

For cell viability assessment, neurospheres were incubated with $20 \mu \mathrm{g} / \mathrm{mL}$ fluorescein diacetate, which stains viable cells, and with $10 \mu \mathrm{g} / \mathrm{mL}$ propidium iodide, a membraneimpermeable DNA dye that stains nonviable cells, in phosphatebuffered saline (PBS) for 5 min, washed with PBS, and observed using fluorescence microscopy (DMI6000; Leica).

\section{Fluorescence microscopy}

Neurospheres were plated on PLOF-coated glass coverslips and allowed to attach for 3 days, fixed in $4 \%$ paraformaldehyde $+4 \%$ sucrose in PBS for $40 \mathrm{~min}$, and processed for immunostaining as described previously. ${ }^{13}$ The antibodies used for population characterization, as well as the secondary antibodies are described in Supplementary Table S1 (Supplementary Data are available online at www.liebertpub.com/tea); cell nuclei were counterstained with TO-PRO-3 (Invitrogen). 
Samples were visualized using fluorescence (DMI6000; Leica) and point-scan confocal (SP5; Leica) microscopy. Merge between channels and maximum z-projections, as well as linear brightness and contrast adjustments of the images were performed using the open-source ImageJ software.

\section{Electron microscopy}

Neurospheres were fixed in $2.5 \%$ glutaraldehyde and $4 \%$ formaldehyde in $0.1 \mathrm{M}$ phosphate buffer ( $\mathrm{pH} 7.4$ ) and then processed for scanning electron microscopy (SEM), transmission electron microscopy (TEM), or serial block-face SEM (SBFSEM). For SEM, samples were dehydrated, critical point dried, mounted on stubs, coated with a thin layer of gold, and imaged with a $6700 \mathrm{~F}$ field emission SEM (JEOL Ltd.). Secondary electron images were collected at $5 \mathrm{keV}$ with a probe current of $10 \mu \mathrm{A}$ and a probe distance of 7.8-7.9 mm. For TEM and SBFSEM, samples were prepared using the National Center for Microscopy and Imaging Research (NCMIR) method. ${ }^{14}$ For TEM, 70-nm sections were collected from neurospheres embedded in Durcupan resin using a UCT ultramicrotome (Leica Microsystems). No poststaining was required due to the density of metal deposited using the NCMIR protocol. Images were acquired using a Tecnai G2 Spirit Biotwin TEM (FEI) and an Orius CCD camera (Gatan). For SBFSEM, neurospheres embedded in Durcupan resin were mounted on a pin and trimmed to a block face of $<1 \mathrm{~mm}^{2}$. Imaging was performed in a Sigma variable-pressure SEM (Carl Zeiss) equipped with a 3 View ultramicrotome (Gatan) for automated serial imaging within the SEM chamber. Images were collected at $4 \mathrm{keV}$ with a pixel dwell time of $3 \mu$ s at $40 \mathrm{~Pa}$. One thousand serial images were collected overnight for each dataset with $7.2 \mathrm{~nm}^{2}$ pixels and a slice thickness of $75 \mathrm{~nm}$. The resulting image stack was aligned using Amira (Visage Imaging, Inc.) and individual cells were manually segmented and rendered using the same software.

\section{Metabolic profiling}

Metabolic profile of neurospheres was assessed at 7Diff and 18Mat and was performed using MM for both cultures, in order to discard the influence of different media composition. Neurospheres were plated on PLOF-coated plates and allowed to attach to the surface. A washing step with PBS was performed before adding fresh MM to the culture. Samples of supernatant were then collected at $6,12,24$, and $48 \mathrm{~h}$ after media exchange and stored at $-20^{\circ} \mathrm{C}$. Neurospheres were harvested and total protein was quantified with Micro BCA Protein Assay Kit (Pierce), according to manufacturer's instructions. Prior to nuclear magnetic resonance (NMR) analysis, samples were thawed and filtered using Vivaspin 500 columns (Sigma-Aldrich) at 14,000 g. To minimize variations in $\mathrm{pH}, 400 \mu \mathrm{L}$ of filtered samples was mixed with $200 \mu \mathrm{L}$ of phosphate buffer $(50 \mathrm{mM}, \mathrm{pH} 7.4)$ with $5 \mathrm{mM} \mathrm{DSS}-\mathrm{d}_{6}$ and centrifuged at $1000 \mathrm{~g}$ for $1 \mathrm{~min}$. For NMR analysis, $500 \mu \mathrm{L}$ of the resulting supernatants were placed into 5-mm NMR tubes. All ${ }^{1} \mathrm{H}$-NMR spectra were recorded at $25^{\circ} \mathrm{C}$ on a Bruker Avance $\mathrm{II}+500 \mathrm{MHz} \mathrm{NMR}$ spectrometer. One-dimensional spectra were recorded using a NOESY-based pulse sequence ( $4 \mathrm{~s}$ acquisition time, $1 \mathrm{~s}$ relaxation time, and $100 \mathrm{~ms}$ mixing time). Typically, 256 scans were collected for each spectrum. All spectra were phase and baseline corrected automatically, with fine adjustments performed manually. Spectra analysis was performed using Chenomx NMR Suite 7.1, using DSS- $\mathrm{d}_{6}$ as internal standard for quantification of metabolites. ${ }^{15}$

\section{Real time quantitative reverse transcription polymerase chain reaction}

Total RNA was extracted with High Pure RNA Isolation Kit (Roche) or RNeasy Mini Kit (Qiagen), according to the manufacturer's instructions. RNA was quantified in a NanoDrop 2000c (Thermo Scientific) and used for cDNA synthesis. Reverse transcription was performed with High Fidelity cDNA Synthesis Kit (Roche), using Anchoredoligo(dT)18 Primer (Roche) or with the Super Script III First Strand synthesis system (Invitrogen), using random hexamers (Invitrogen). Real time quantitative reverse transcription polymerase chain reaction (qRT-PCR) were performed in triplicates using LightCycler 480 SYBR Green I Master Kit (Roche) and primers were listed in Supplementary Table S2. As alternative, TaqMan Universal PCR Master Mix (Applied Biosystems) and TaqMan ${ }^{\circledR}$ Gene Expression Assays (Applied Biosystems) listed in Supplementary Table S3 were used. Reactions were performed with Applied Biosystems 7300 Real Time PCR system or LightCycler 480 Instrument II 96-well block (Roche). Quantification of cycle values and melting curves was determined using LightCycler 480 Software version 1.5 (Roche). All data were analyzed using the $2^{-\Delta \Delta \mathrm{Ct}}$ method for relative gene expression analysis. ${ }^{16}$ Changes in gene expression were normalized using the housekeeping gene RPL22 (ribosomal protein L22) as internal control.

\section{Microarray analysis}

Three independent biological replicates of both undifferentiated and differentiated samples were analyzed by using the Affymetrix HG U133 plus2 gene chips, interrogating more than 47,000 transcripts. Microarray data were normalized by the $\log$ scale robust multiarray analysis procedure using $\mathrm{R}$ (Bioconductor) and differentially expressed genes were obtained with limma package. ${ }^{17} \mathrm{~A}$ moderated $t$-test was performed between differentiated and undifferentiated groups selecting genes with a false discovery rate (FDR) value $\leq 0.01$ and with $\geq 2$-fold change for upregulated genes and $\leq 2$-fold change for downregulated genes.

The identified genes were categorized in generic gene ontology (GO) functional clusters using Cytoscape_v 2.8.3 and its plug-in BINGO 2.44 (Biological Networks Gene Ontology tool). ${ }^{18}$ The significance of overrepresented GO categories was assessed with a hypergeometric test and the Benjamini and Hochberg FDR correction. A corrected $p$-value $<0.05$ was considered significant and only significantly overrepresented GO categories are presented. ${ }^{19}$

\section{Western blot}

Cells were lysed in lysis buffer $(50 \mathrm{mM}$ Tris, $5 \mathrm{mM}$ ethylenediaminetetraaceticacid, $150 \mathrm{mM} \mathrm{NaCl}$, and $1 \%$ Triton $\mathrm{X}-100$ [pH 7.4]) for $30 \mathrm{~min}$ at $4^{\circ} \mathrm{C}$. Extracts were clarified by centrifugation at $15,000 \mathrm{~g}$ for $10 \mathrm{~min}$, followed by protein precipitation by overnight incubation in $80 \%$ ethanol. Precipitated proteins were collected at $15,000 \mathrm{~g}$ for $15 \mathrm{~min}$ and 
solubilized in reducing NuPAGE sample buffer (Invitrogen). Total protein was quantified with Micro BCA Protein Assay Kit (Pierce). Protein extracts were resolved on a $1 \mathrm{~mm}$ NuPAGE $^{\circledR}$ Novex BisTris gel (Invitrogen) under reducing conditions and transferred with iBlot system (Invitrogen), according to the manufacturer's instructions. Membranes were blocked by incubation for $1 \mathrm{~h}$ with blocking solution (0.1\% Tween 20 and 5\% dry milk in PBS), and incubated overnight with primary antibody (Supplementary Table S1) diluted in blocking solution. Blots were developed using the enhanced chemiluminescence (ECL) detection system after incubation for $1 \mathrm{~h}$ at room temperature with horseradish-peroxidase-labeled anti-mouse immunoglobulin $\mathrm{G}$ or anti-rabbit antibody (GE Healthcare) at 1:5000 dilution. Chemiluminescence detection was performed by incubating the membranes with Amersham ECL Prime western blotting detection reagent (GE Healthcare) and analyzed under ChemiDoc XRS System (Bio-Rad).

\section{Synaptic vesicle trafficking}

Neurospheres plated on PLOF-coated glass coverslips were washed with PBS and exposed to a high-potassiumdepolarizing solution $(100 \mathrm{mM} \mathrm{KCl}$ buffer; Supplementary Table S4), for $5 \mathrm{~min}$. Afterward, neurospheres were incubated with $10 \mu \mathrm{M}$ FM 1-43 dye (Invitrogen) dissolved in normal saline (5 mM KCl buffer; Supplementary Table S4) for 15 min and washed with ADVASEP-7 (Sigma) dissolved in $5 \mathrm{mM} \mathrm{KCl}$ buffer for $1 \mathrm{~min}$. This was followed by three washes of $1 \mathrm{~min}$ with $5 \mathrm{mM} \mathrm{KCl}$ buffer prior to imaging. Exocytosis was stimulated with $100 \mathrm{mM} \mathrm{KCl}$ buffer and samples were imaged live in a fluorescence microscope (DMI6000; Leica) to monitor synaptic vesicle release. Fluorescence intensity was measured using ImageJ.

\section{Calcium assay}

Neurospheres were incubated with $1 \times$ Fluo4 Direct calcium reagent (Invitrogen) for $30 \mathrm{~min}$ at $37^{\circ} \mathrm{C}, 5 \% \mathrm{CO}_{2}$, and $3 \% \mathrm{O}_{2}$ and for $15 \mathrm{~min}$ at room temperature. Samples were then imaged live using spinning-disk microscopy (Nikon Eclipse Ti-E, confocal scanner: Yokogawa CSU-x1). Fluorescence change over time is defined as $\Delta F / F_{0}=\left(F-F_{0}\right) / F_{0}$, where $F$ is the fluorescence at any time point, and $F_{0}$ the baseline fluorescence determined by baseline fitting across the whole movie for each cell using PeakFit Software (v4.12).

\section{Neurotransmitter release and quantification}

Neurotransmitter synthesis and release was assessed at 18Mat. Neurospheres plated on PLOF-coated glass coverslips were washed with PBS and exposed to a high-potassiumdepolarizing solution $(100 \mathrm{mM} \mathrm{KCl}$ buffer; Supplementary Table S4) for $15 \mathrm{~min}$. The obtained supernatant was then collected and stored at $-20^{\circ} \mathrm{C}$ prior to high-performance liquid chromatography (HPLC) analysis. Dopamine in cell supernatants was quantified by HPLC after sample precipitation with $10 \%$ perchloric acid (8:1). The separated monoamines were detected by fluorescence ${ }^{20}$ and quantified by comparison to a calibration curve of dopamine. GABA was quantified by HPLC after supernatant freeze drying, using a precolumn derivatization method (Waters AccQ.Tag Amino Acid Analysis) previously described. ${ }^{21}$

\section{Electrophysiology recording}

Neurospheres were visualized in an inverted phasecontrast microscope. Whole-cell voltage-clamp recordings were made from neurons within differentiated neurospheres at room temperature using an Axopatch 200B (Axon Instruments, Inc.). Microelectrode contact to individual cells was made visually (cells on the neurosphere surface) or in deeper layers, using the blind approach common in brain slice recordings. ${ }^{22}$ Microelectrodes (1.2-3.0 M $\Omega$ ) were pulled from borosilicate glass (Science Products $\mathrm{GmbH}$ ). Two sets of solutions were used: one to record voltage-activated $\mathrm{K}^{+}$ conductances and to estimate membrane potential $\left(V_{\mathrm{m}}\right.$, under current clamp) (set 1), and a second to record excitatory ligand-activated currents (set 2). For set 1, electrodes were filled with solution containing (in $\mathrm{mM}$ ) $\mathrm{KMeSO}_{4}$ (140), $\mathrm{MgCl}_{2}$ (1), HEPES (10), EGTA (10), $\mathrm{CaCl}_{2}$ (1), $\mathrm{Na}_{2} \mathrm{ATP}$ (2), and Na-GTP (0.4) ( $\mathrm{pH} \mathrm{7.2-7.3)} \mathrm{and} \mathrm{titrated} \mathrm{with} \mathrm{KOH}$ (calculated free $\left[\mathrm{Ca}^{2+}\right]=60 \mathrm{nM}$ by Webmaxclite $\mathrm{v} 1.15$; MaxChelator), and external solution to record outward $\mathrm{K}^{+}$ currents contained (in $\mathrm{mM}$ ) $\mathrm{NaCl}$ (135), $\mathrm{KCl}$ (5.4), $\mathrm{CaCl}_{2}$ (2), $\mathrm{MgCl}_{2}$ (1.5), HEPES (10), and D-glucose (25) (pH 7.4) and titrated with $\mathrm{NaOH}$. In set 2, the pipette solution contained (in $\mathrm{mM}$ ) $\mathrm{CsF}$ (140), $\mathrm{NaCl}$ (10), HEPES (10), and EGTA (5) (pH 7.3) and titrated with $\mathrm{CsOH}$, and external solution contained (in $\mathrm{mM}$ ) $\mathrm{NaCl}$ (100), $\mathrm{KCl}$ (5), HEPES (10), $\mathrm{CaCl}_{2}$ (1.8), $\mathrm{MgCl}_{2}$ (1), TEA-Cl (24), 4-amino-pyridine (3), and D-glucose (23) ( $\mathrm{pH} 7.4)$ and titrated with $\mathrm{NaOH}$. External bathing solution was constantly superfused $(\sim 2-$ $3 \mathrm{~mL} / \mathrm{min})$.

The junction potentials estimated on JPCalc software (v2) for the external solution for set 1 of solutions was -8.8 and $-8.5 \mathrm{mV}$ for set 2 of solutions; data were not corrected for the junction potential. Currents were measured with cell capacitance compensation and series resistance compensation (80\%), filtered at $2 \mathrm{kHz}$, sampled at $5 \mathrm{kHz}$, using a Digidata 1200 ADC converter (Axon Instruments) and pClamp software (v6). Time was allowed for the stabilization of the recording before experiments were conducted.

Different voltage-clamp protocols were applied according to the experimental needs, as follows: (1) to study outward currents: a single command pulse to a fixed voltage was used and, to isolate the fast-current component, such command pulse was preceded by a pulse to -120 or to $-30 \mathrm{mV}$. Sets of incremental depolarizing commands were used for the characterization of the voltage dependence of activation. In this protocol, leakage current was compensated a posteriori from the current-voltage relation generated by a set of 12 prepulses in increments of $2 \mathrm{mV}$ from $-75 \mathrm{mV}$. To study steady-state inactivation, a single-command step was preceded by incremental prepulses. ${ }^{23}$ L-Glutamate-induced currents were obtained by continuous recording under variable resting potentials. Glutamate was administered with a handmade adaptation of the perfusion system using gravity. Details of all the voltage protocols are given in the "Results" section.

Whole-cell data were analyzed using Clampfit (v9) (Axon Instruments, Inc.), Pulsefit (v8.67), and Origin (v5) (Microcal Origin). Outward currents were measured as illustrated previously. ${ }^{23}$ Peak current was taken for current amplitude of the faster current component; for each experiment, current decay was best fit with a sum of two exponentials 
(Eq. 3). For each current sweep, the amplitude of the slower component was taken at a time equal to $5 \times \tau_{\text {fast }}$ from the start of the command pulse. For steady-state inactivation and activation profiles, current values were fit with the following Boltzmann equation:

$$
I=A_{1}-A_{2} /\left\{1+\exp \left[\left(V_{1 / 2}-V\right) / V_{s}\right]\right\}+A_{2},
$$

where $I$ is the current amplitude at the test potential $V, V_{1 / 2}$ is the half-activation potential, $V_{\mathrm{s}}$ is the slope constant, and $A_{1}$ and $A_{2}$ are coefficients.

In the case of voltage dependence of activation, data were converted to conductance using the relationship $G=I /(V-$ $\left.E_{\mathrm{K}+}\right)$, where $I$ is current amplitude, $V$ is the step command potential, and $E_{\mathrm{K}+}$ the estimated equilibrium potential for $\mathrm{K}^{+}$. Results were plotted against the step command potential and fit with the following equation:

$$
G=A_{1}-A_{2} /\left\{1+\exp \left[\left(V-V_{1 / 2}\right) / V_{\mathrm{s}}\right]\right\}+A_{2}
$$

In the vast majority of the cases, current relaxations required the sum of two exponentials, using the following equation:

$$
F(t)=A_{\mathrm{f}} \exp \left(-t / \tau_{\text {fast }}\right)+A_{\mathrm{s}} \exp \left(-t / \tau_{\text {slow }}\right)+C,
$$

where $\tau_{\text {fast }}$ and $\tau_{\text {slow }}$ are the time constants of the fast and slow inactivating components, respectively; $A_{\text {fast }}$ and $A_{\text {slow }}$ are the respective coefficients; and $C$ is a constant.

\section{Statistical analysis}

Data are expressed as the mean \pm standard error of the mean. Data were analyzed using GraphPad Prism (version 5.01) by an analysis of variance, followed by Tukey's post-hoc multiple-comparison test, and for metabolic profiling data using a two-tailed $t$-test. The $\alpha$ value was set at 0.05 with a $95 \%$ confidence interval and statistical significance was defined based on $p$-value $(* * * p<0.001, * * p<0.01$, and $* p<0.05)$.

\section{Results}

hmNPC 3D differentiation induces molecular, metabolic, and morphological remodeling

To determine the potential of hmNPCs to generate innovative cellular models for CNS diseases, we assessed the cellular changes induced by 3D culture conditions. For multipotent hmNPC differentiation, cells expanded in twodimensional (2D) cultures were kept in suspension culture systems with constant stirring (Fig. 1A). As recently described by our group, ${ }^{10} \mathrm{hmNPCs}$ efficiently organized into neurospheres, resulting in a homogenous culture in terms of neurosphere size, ranging between 300 and $400 \mu \mathrm{m}$, as well as high cell viability (Supplementary Fig. S1A, B).

The onset of differentiation led to a significant downregulation (up to 4-fold) in the expression of the DNA polymerase cofactor PCNA (Fig. 1B), which was maintained during 32 days of differentiation, suggesting a decrease in cell proliferation. Concomitantly, the neurotrophic receptors TrkA and TrkB were positively modulated upon differentiation with a 2 - and 28 -fold increase at $18 \mathrm{Mat}$, respectively (Fig. 1B). The expression of these tyrosine kinase
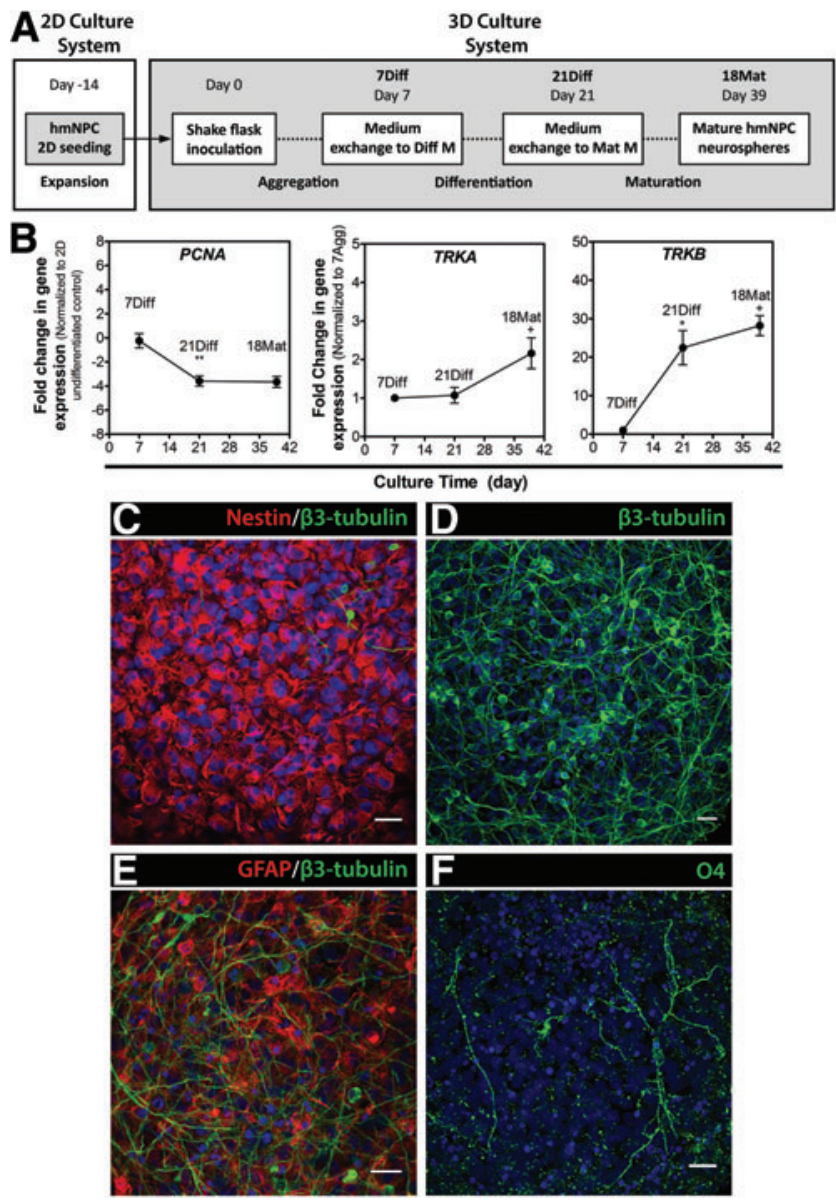

FIG. 1. Differentiation and cell population dynamics of hmNPCs as 3D neurospheres. (A) Workflow of expansion and 3D differentiation of hmNPC cultures (Diff M, differentiation medium; Mat M, maturation medium). (B) qRTPCR analysis of PCNA, TRKA, and TRKB gene expression. Fold changes in gene expression normalized to undifferentiated hmNPCs expanded in 2D culture systems $(P C N A)$ or to 7Diff (TRKA and TRKB). Data are mean \pm SEM of three independent cultures; asterisks indicate significant difference $\left({ }^{*} p<0.05, * * p<0.01\right.$ vs. 7 Diff; ${ }^{+} p<0.05$ vs. 21 Diff $)$ by a one-way ANOVA analysis with a Tukey's post-hoc multiple-comparison test. (C-F) Confocal immunofluorescence microscopy of whole neurospheres from 7Diff (C) and 18Mat (D-F). Maximum-intensity z-projections of 22 (C), 7 (D), 5 (E), and 25 (F) optical sections of $0.5 \mu \mathrm{m}$. Scale bars $=20 \mu \mathrm{m}$. Detection of nestin, $\beta 3$-tubulin, GFAP, and O4; nuclei were labeled with TO-PRO-3. 2D, two-dimensional; 3D, three-dimensional; 7Diff, 7 days in aggregation medium; 21Diff, 14 days in differentiation medium; 18Mat, 18 days in maturation medium; hmNPCs, human midbrain-derived neural progenitor cells; SEM, standard error of the mean; ANOVA, analysis of variance. Color images available online at www.liebertpub.com/tea

receptors, activated by different neurotrophic factors, has been correlated with midbrain dopaminergic neurons during pre- and postnatal development, ${ }^{24,25}$ with reports suggesting an increased dependence on TrkB/BDNF signaling with differentiation. ${ }^{26}$

In contrast to 7Diff, when cells presented predominantly a progenitor phenotype, expressing the early neuroepithelial 
marker nestin (Fig. 1C), by 18Mat, a dense neurite network of $\beta 3$-tubulin-positive neurons was observed both at the surface and inside the neurospheres (Fig. 1D). Moreover, differentiation into the three neural lineages was observed, as cells positive for the astrocytic-lineage marker glial fibrillary acidic protein (GFAP) and oligodendrocytic-lineage O4 were also detected (Fig. 1E, F).

Morphological changes, including membrane rearrangements and cytoplasmic volume reduction, were observed during the differentiation process (Fig. 2; Supplementary Videos S1 and $\mathrm{S} 2$ ), with a transition from lamellipodia, which were abundant in early differentiation stages (Fig. 2A, C), toward filopodia (Fig. 2B, D). In parallel, the complexity of cell processes and arborization of the network increased (Fig. 2E, F), with thin filopodia $(0.2-0.5 \mu \mathrm{m})$ protruding from the cell processes (Fig. $2 \mathrm{H}$ ), which may indicate the ability of differentiating neurons to undergo
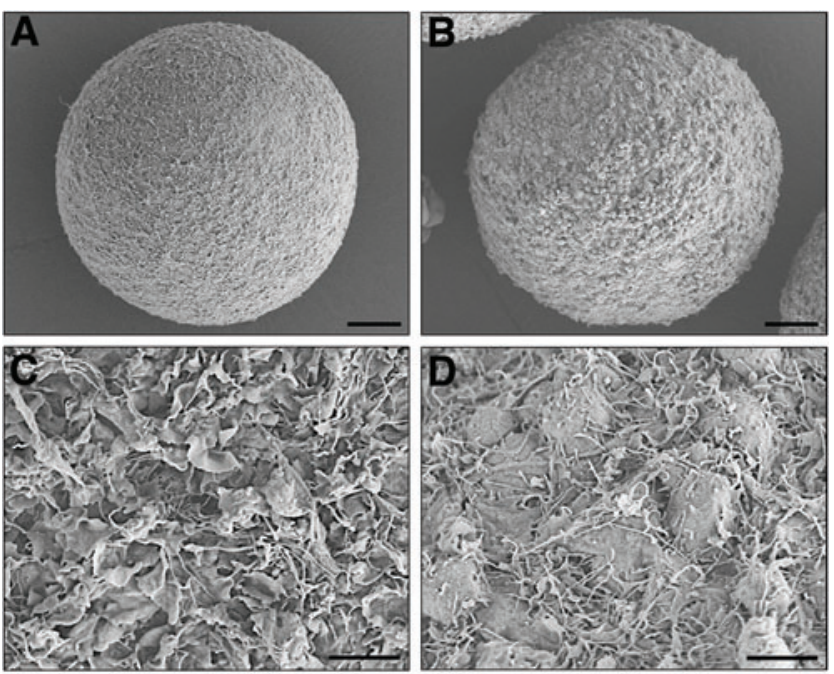

E
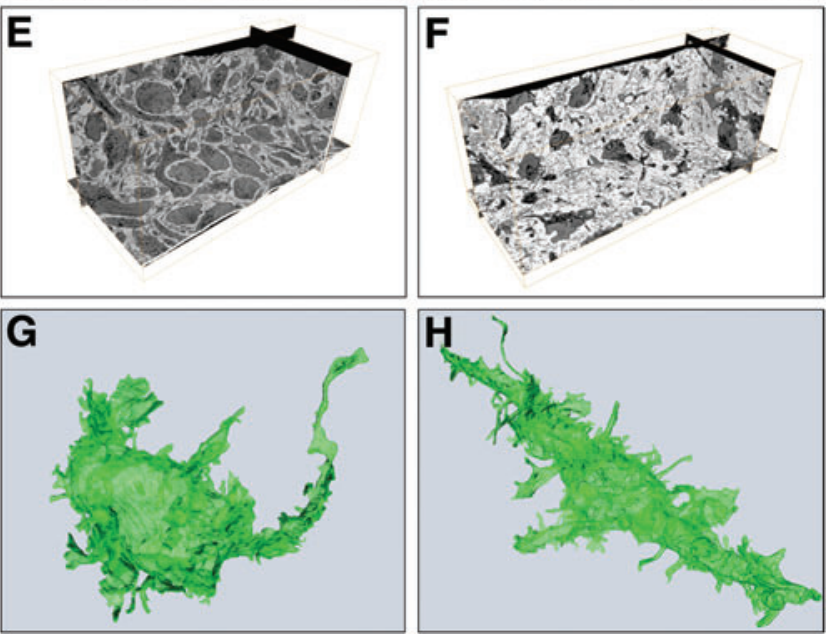

FIG. 2. Morphological characterization of hmNPC neurospheres along differentiation. (A-D) Scanning electron microscopy of neurospheres from 7Diff $(\mathbf{A}, \mathbf{C})$ and 21Diff (B, D). (E, F) SBFSEM of neurospheres from 7Diff (E) and 21Diff $(\mathbf{F})$. (G, H) Single-cell reconstruction obtained from SBFSEM data from 7Diff $(\mathbf{G})$ and 21Diff $(\mathbf{H})$. Scale bars $=50 \mu \mathrm{m}(\mathbf{A}, \mathbf{B})$ and $5 \mu \mathrm{m}(\mathbf{C}, \mathbf{D})$. SBFSEM, serial block-face scanning electron microscopy. Color images available online at www.liebertpub.com/tea synaptogenesis and form dendritic spines. ${ }^{27,28}$ Putative synaptic precursor sites were already visible in 7Diff neurospheres (Fig. $3 \mathrm{D})$, suggesting that the synaptic machinery might be activated at early differentiation stages. Ultrastructural examination revealed intact mitochondria, Golgi apparatus, and endoplasmic reticulum both in undifferentiated (7Diff, Fig. 3C) and differentiated cultures (21Diff and 18Mat, Fig. 3G, K respectively), consistent with the cells within the neurospheres being healthy
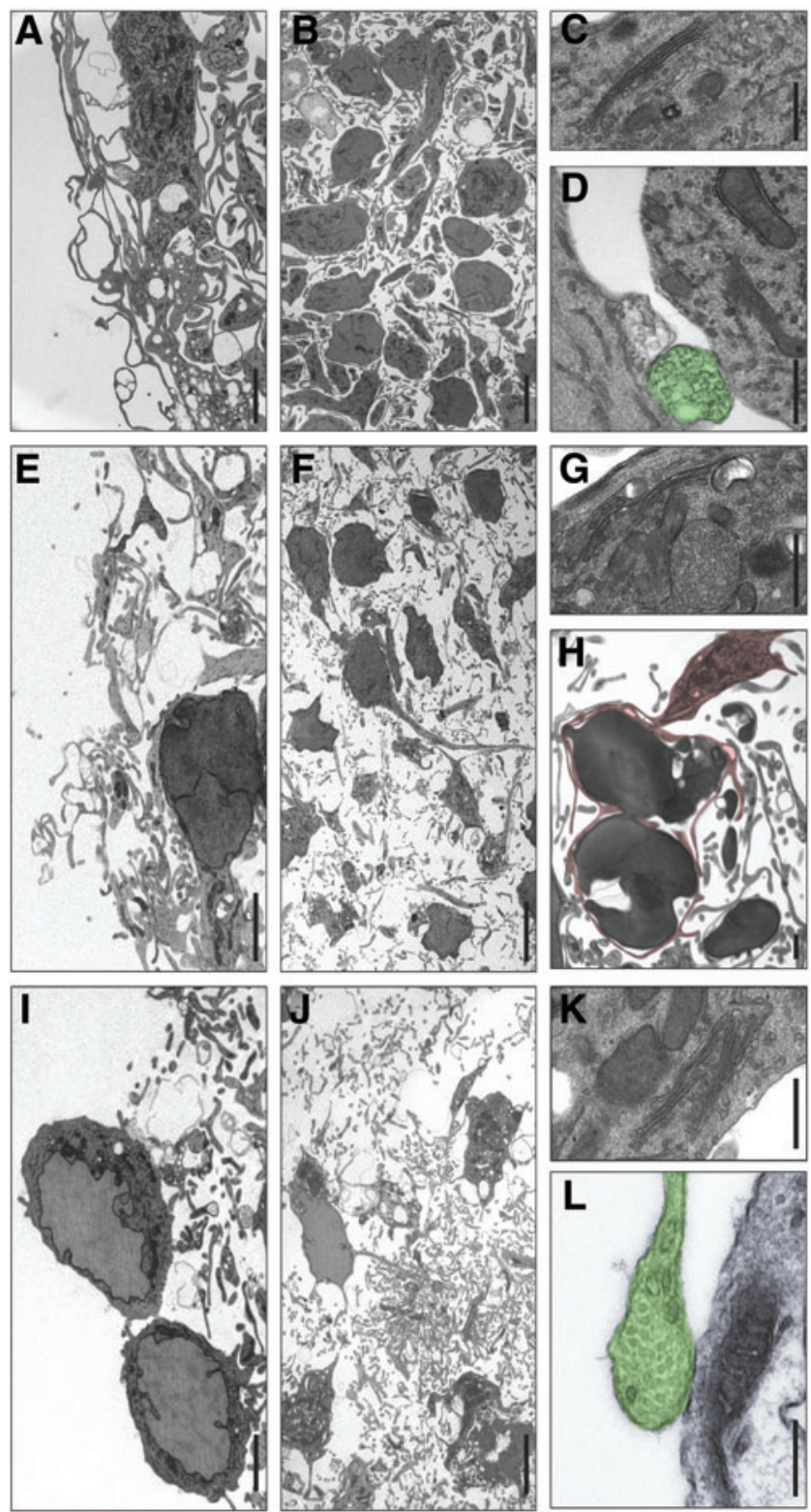

FIG. 3. Ultrastructural characterization of hmNPC neurospheres along differentiation. Transmission electron microscopy of external $(\mathbf{A}, \mathbf{E}, \mathbf{I})$ and inner layers $(\mathbf{B}, \mathbf{F}, \mathbf{J})$ of hmNPC neurospheres at day 7Diff $(\mathbf{A}, \mathbf{B}), 21 \operatorname{Diff}(\mathbf{E}, \mathbf{F})$, and 18Mat (I, J). Inset of a cell depicting Golgi apparatus at 7Diff (C), 21Diff (G), and 18Mat (K). (D) Putative site of synaptogenesis at 7Diff (in green). (H) Oligodendrocytelike cell at 21Diff (in red). (L) Putative synaptic site at $18 \mathrm{Mat}$ (in green). Scale bars $=2 \mu \mathrm{m}(\mathbf{A}, \mathbf{E}, \mathbf{I}), 5 \mu \mathrm{m}(\mathbf{B}, \mathbf{F}, \mathbf{J})$, $0.5 \mu \mathrm{m}(\mathbf{C}, \mathbf{D}, \mathbf{G}, \mathbf{H}, \mathbf{K})$, and $0.2 \mu \mathrm{m}(\mathbf{H})$. Color images available online at www.liebertpub.com/tea 
and metabolically active. These features were observed throughout the neurospheres (for 7Diff, 21Diff, and 18Mat; Fig. 3A, B and E, F and I, J, respectively), which lacked any obvious sign of a necrotic center (Supplementary Videos S1-S3). Interestingly, in differentiated cultures (21Diff), it was also possible to identify cells with morphological features typical of oligodendrocytes, enfolding neighboring cells with membrane protrusions (Fig. 3H). By 18Mat putative synaptic sites enriched in synaptic vesicles and contacting adjacent cells were identified (Fig. 3L).

Aiming at assessing metabolic alterations along differentiation, ${ }^{1} \mathrm{H}-\mathrm{NMR}$ metabolic profiling of cell supernatants along $48 \mathrm{~h}$ of culture was determined for undifferentiated and differentiated hmNPC neurospheres (Fig. 4; Supplementary Fig. S2). 7Diff neurospheres presented high-glucose consumption to lactate production ratio $\left(Y_{\mathrm{Lac} / \mathrm{Glc}}=1.68 \pm 0.22\right)$, indicating anaerobic glucose utilization in progenitor cells. Despite taking up glucose at lower rates (Fig. 4B), differentiated hmNPC neurospheres maintained the reliance on glycolytic metabolism $\left(Y_{\mathrm{Lac} / \mathrm{Glc}}=1.91 \pm 0.12\right)$. As for pyruvate uptake and alanine accumulation rates, a 4-fold increase and decrease, respectively, were observed (Fig. 4B), suggesting changes during differentiation in the alanine-lactate shuttle. Although glutamate was not detected in hmNPC cultures, its cyclized form pyroglutamate was released at higher rates in differentiated cultures (Fig. 4A, B). Conversely, the accumulation rates of 2-oxoisocaproate and methylsuccinate, metabolites that result from the metabolism of branched- chain amino acids (BCAAs), were maintained in undifferentiated and differentiated cultures.

\section{$3 D$ differentiation activates neurogenesis developmental pathways}

A coordinated change in gene expression is a hallmark of cellular differentiation. We therefore decided to study the transcriptomic programs that were modulated during hmNPC neurosphere differentiation. Global transcriptional analysis was performed in differentiated neurospheres (21Diff) and compared with undifferentiated hmNPCs (Fig. 5A). The gene expression profile showed that 807 probes, which corresponded to 664 unique genes, were differently modulated upon neurosphere differentiation.

$\mathrm{GO}$ analysis showed that the $3 \mathrm{D}$ differentiation process led to a significant enrichment in genes involved in cell cycle, cell differentiation, cytoskeleton organization, and proteinaceous ECM (Fig. 5B). Genes involved in the cell cycle progression, such as MKI67, cyclin A2, cyclin B1, and cyclin D1, and mitotic genes like $B U B 1 B, B U B 1, C C N B 1, C D C 25 A, C D K 1, A U R K A$, $K I F 23, M A D 2 L 1$, and BIRC5 were significantly downregulated after 3D differentiation, suggesting an increase in cells exiting the cell cycle. Additionally, markers of neural stem cells, such as SPRY1, SPRY2, and FBXO5, were also downregulated, whereas genes involved in neurogenesis and neuronal metabolism, namely, NOTCH2, PAX6, PRDM16, NR4A2, PDE3A, DCLK1, SCG2, PAQRB, EFNA5, MAPT, APOE, and MAL,
FIG. 4. Metabolic profiling of hmNPC neurospheres along differentiation. (A) Concentration profiles of the main metabolites quantified in the exometabolome of hmNPC cultures at 7Diff (black circles) and 18Mat (white circles) that have significantly changed during $48 \mathrm{~h}$. Data from one representative experiment of two independent experiments. (B) Specific rates $\left(\mathrm{nmol} \cdot \mathrm{mg}\right.$ protein $\left.{ }^{-1} \cdot \mathrm{h}^{-1}\right)$ determined at 7Diff and 18Mat. Data are mean \pm SEM of regression analysis performed with all time points from two independent experiments, with two replicates each. Asterisks indicate significant difference $(* p<0.05$ and $* * p<0.01)$ by a two-tailed $t$-test.
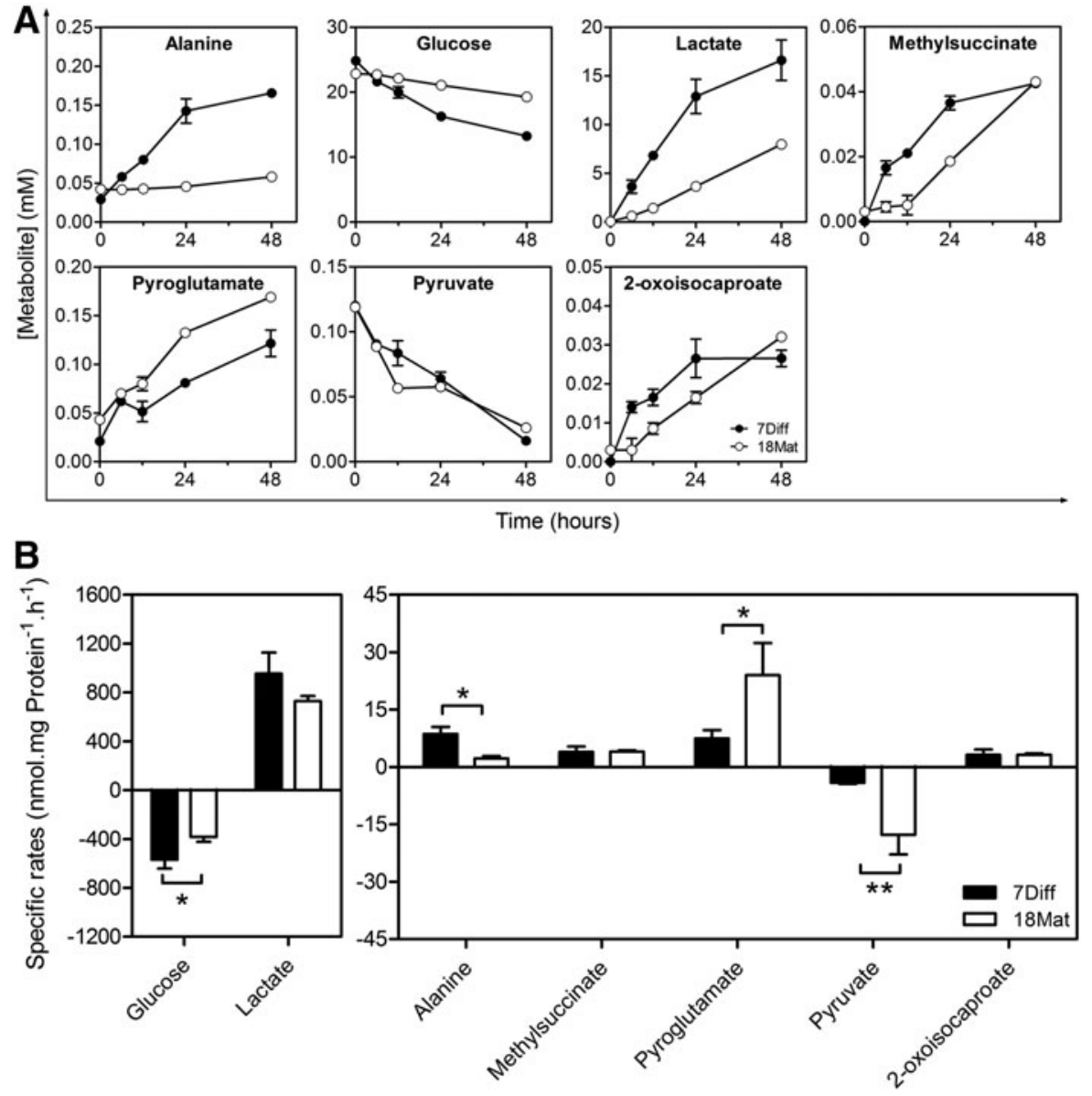

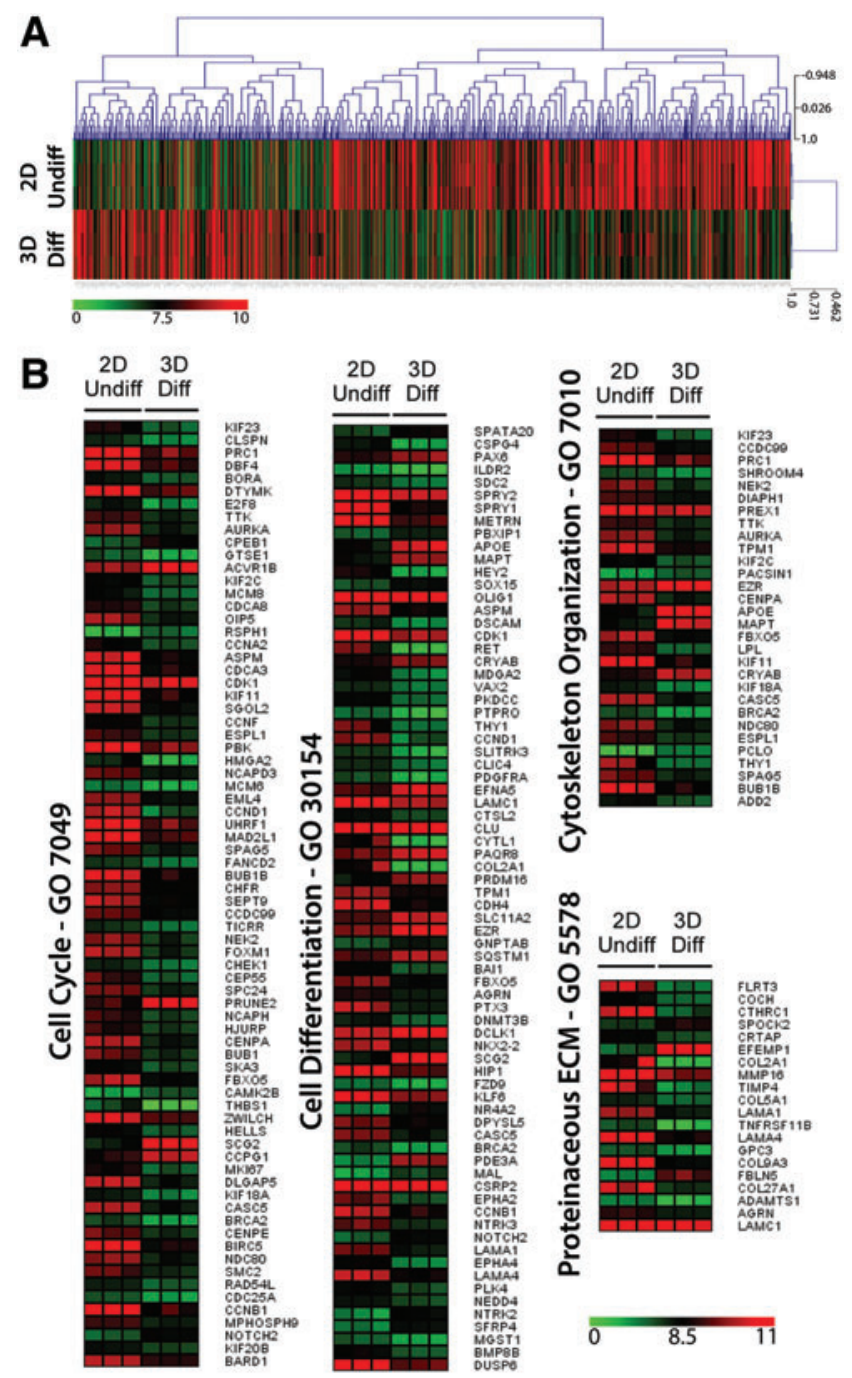

FIG. 5. Global gene expression analysis of differentiated hmNPC neurospheres. (A) Hierarchical clustering of the levels of modulation of 807 probes, corresponding to 664 unique genes, significantly altered (FDR $\leq 0.01,2$-fold change in upregulated and downregulated) compared with undifferentiated cells (data of three independent cultures; each column represents an independent sample). Color bar represents the scale of RMA-normalized log2-transformed expression value of each transcript of the replicates. (B) Heat maps of the expression patterns (RMA normalized values) of genes altered in each replicate of four significant gene ontology categories (corrected $p$-value $<0.05$ ) according to a color scale ranging from green to red. Enriched biological clusters consist in cell cycle (FDR $\left.4.2 \times 10^{-18}\right)$, cell differentiation $\left(3.2 \times 10^{-4}\right)$, proteinaceous extracellular matrix $\left(6.9 \times 10^{-3}\right)$, and cytoskeleton organization $\left(2.58 \times 10^{-3}\right)$. FDR, false discovery rate; RMA, robust multi-array average. Color images available online at www .liebertpub.com/tea

were increased. The activation of TGF $\beta 1$-signaling-associated genes, such as $A C V R 1 B$ or PRUNE2, which are involved in the maintenance of mature $\mathrm{CNS},{ }^{29}$ suggests maturation of the neuronal population. Several ECM-associated genes were downregulated after differentiation, such as genes involved in collagen synthesis and binding (COL2A1, COL5A1, COL9A3, COL27A1, CTHRC1, and CRTAP), laminin synthesis
(LAMA1 and LAMA4), and fibronectin binding (FLRT3). Concomitantly, an upregulation of SPOCK2, EFEMP1, and $F B L N 5$ genes, which code for proteins involved in glycosaminoglycan binding and proteoglycans, ${ }^{30}$ was observed. Together these results suggested a significant remodeling of the ECM composition in the 3D environment of the differentiated neurospheres toward a higher similarity to the in vivo neural ECM composition. ${ }^{31}$

\section{Extended $3 D$ differentiation enhances dopaminergic phenotype}

Neuronal differentiation toward the dopaminergic lineage depends on specific developmental programs that rely on the sequential activation of specific transcription factors. At 21Diff, an increase in the expression levels of NURRl, a transcription factor critical for the development of the dopaminergic phenotype; tyrosine hydroxylase $(\mathrm{TH})$, the ratelimiting enzyme in dopamine synthesis; and dopamine receptor D2 (DRD2) indicated the activation of genetic pathways controlling the dopaminergic phenotype (Fig. 6A-C). Extending the differentiation process for additional 18 days in presence of cAMP led to a significant upregulation of these markers (10-, 450-, and 4-fold increase at 18Mat relatively to day 7, for $N U R R 1, T H$, and DRD2, respectively) (Fig. 6A-C). Moreover, the increase in TH protein levels along differentiation (Fig. 6D) and detection of cells positive for TH (Fig. $6 \mathrm{E}$ ), as well as dopaminergic transcription factor Pitx3 (Fig. $6 \mathrm{~F})$, at 18Mat further suggested the establishment and maintenance of the dopaminergic phenotype.

Along with the expression of dopaminergic markers, a significant upregulation of the presynaptic vesicular GABA transporter (vGAT) was observed (Supplementary Fig. S3A), suggesting the coexistence of dopaminergic and GABAergic neuronal populations.

Altogether, our results indicated that hmNPC neurospheres retained their midbrain developmental patterns by expressing key dopaminergic markers upon differentiation, and revealed the importance of extending the differentiation process by 18 days to increase the neuronal differentiation efficiency.

\section{Neuronal maturation and synaptic functionality}

In addition to the increased expression of key lineagespecific markers, it was essential to demonstrate the functional properties of the differentiated cells derived from hmNPC neurospheres. To assess neuronal synaptic maturation, we analyzed the expression of proteins involved in synaptic formation and homeostasis. Expression of different presynaptic markers, such as synapsin II (SYN2), synaptophysin $(S Y P)$, and synaptotagmin I (SYT1), gradually increased during differentiation, reaching up to 5-, 2-, and 6-fold greater levels by the end of the differentiation process (Fig. 7A). Additionally, synaptophysin, an integral presynaptic vesicle glycoprotein, was detected in a typical punctate pattern across the differentiated neurospheres (Fig. 7B). These results together with the ultrastructural evidence (Fig. 2) suggest that presynaptic components cluster in differentiated hmNPC neurospheres into synaptic vesicle-like organelles. The 1.5-fold increase in postsynaptic density protein 95 (PSD95) at 18Mat suggested the accumulation of postsynaptic markers in these cultures (Fig. 7A). 

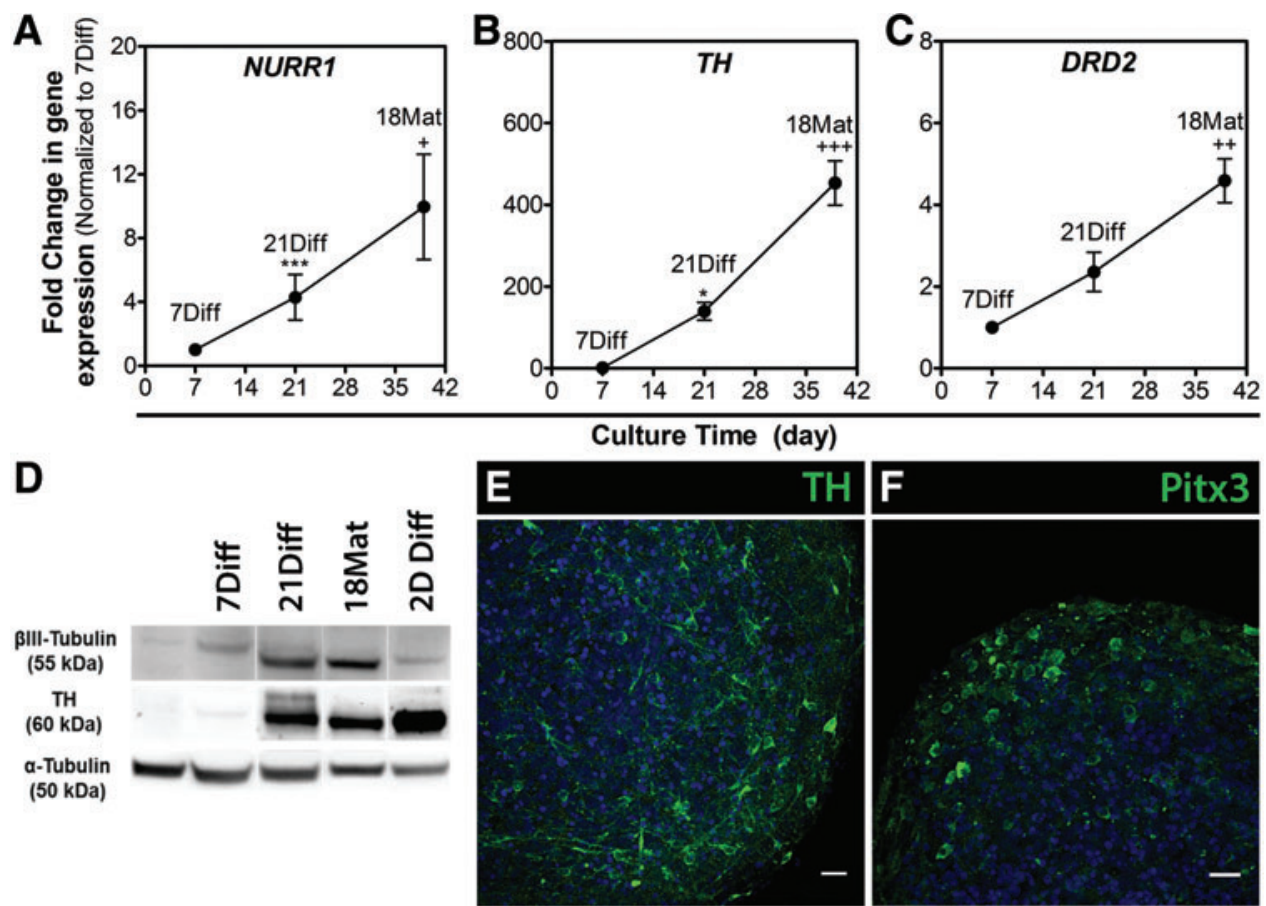

FIG. 6. Dopaminergic differentiation of hmNPC neurospheres. (A-C) qRT-PCR analysis of $N U R R 1$ (A), TH (B), and DRD2 (C) gene expression. Fold changes in gene expression were normalized to 7Diff. Data are mean \pm SEM of three independent cultures; asterisks indicate significant difference $\left({ }^{*} p<0.05,{ }^{* * *} p<0.001\right.$ vs. $7 \mathrm{Diff} ;{ }^{+} p<0.05,{ }^{++} p<0.01$, ${ }^{+++} p<0.001$ vs. 21Diff) by a one-way ANOVA analysis with a Tukey's post-hoc multiple-comparison test. (D) Western blot analysis of undifferentiated hmNPCs and neurospheres along differentiation. $\alpha$-Tubulin detection was used as loading control. Data from one representative experiment of three independent experiments. P0 - undifferentiated hmNPCs expanded in 2D culture systems. (E, F) Confocal immunofluorescence microscopy of whole neurospheres from $18 \mathrm{Mat}$ Maximum-intensity z-projections of $5(\mathbf{E})$ and $56(\mathbf{F})$ optical sections of $0.5(\mathbf{E})$ and $0.33 \mu \mathrm{m}(\mathbf{F})$. Detection of TH and Pitx3; nuclei were labeled with TO-PRO-3. Scale bar $=20 \mu \mathrm{m}$. Color images available online at www.liebertpub.com/tea

Synaptic activity was assessed using the fluorescent probe FM1-43, ${ }^{32,33}$ by the ability of differentiated neurospheres to respond to depolarizing stimuli. Neurospheres at different stages of differentiation were loaded with FM1-43, and their destaining kinetics upon depolarization indicated that the same depolarizing stimuli led to a modest decrease in the fluorescence intensity of 7Diff and 21Diff neurospheres, when compared with neurospheres at the end of the differentiation process (18Mat), in which fluorescence dropped to less than half of its initial value (Fig. 7D). These results suggested that differentiated neurospheres showed a higher number of mature neurons with functional synaptic terminals, which were able to respond to depolarizing stimuli.

To further assess neuronal functionality, Fluo-4-based $\mathrm{Ca}^{2+}$ imaging studies were performed in 18Mat neurospheres (Fig. 7C; Supplementary Video S4). A variety of spontaneous firing patterns identified in individual cells may suggest the presence of both neurons and astrocytes, since the latter can also present spontaneous $\mathrm{Ca}^{2+}$ transients, although with significant lower frequencies compared with neurons. ${ }^{34,35}$

Moreover, an important physiological property of dopaminergic neurons is their ability to produce and release dopamine in response to a depolarizing stimulus. Differentiated neurospheres (18Mat) in presence of $100 \mathrm{mM} \mathrm{KCl}$ were able to respond, releasing $252 \mathrm{ng}$ of dopamine per $\mathrm{mg}$ of total protein (Fig. 7E), further indicating a mature phe- notype of the dopaminergic neurons in culture. In agreement with observation of upregulation of GABAergic marker vGAT, differentiated hmNPC neurospheres were also able to synthesize and release GABA (Supplementary Fig. S3B), in a $\mathrm{KCl}$-dependent response, further suggesting the presence of mature GABAergic neurons.

\section{Voltage-activated and glutamate-evoked currents}

Functionality of neurons within differentiated neurospheres was further assessed by whole-cell voltage-clamp recordings. Cells exhibited hyperpolarized membrane potential values $\left(V_{\mathrm{m}}=-70.1 \pm 0.3 \mathrm{mV}, n=9\right)$, indicating a high level of polarity. Voltage-activated potassium currents recorded at physiological $\mathrm{K}^{+}$concentrations generated typical neuronal outward currents (Fig. 8A). The outward current following the prepulse to $-120 \mathrm{mV}$ comprised two major components, $I_{\text {fast }}$ and $I_{\text {slow }}$, which were fit with a sum of two exponentials to determine time constants of $25.9 \pm 0.9 \mathrm{~ms}(n=9)\left(\tau_{\text {fast }}\right)$ and $234.4 \pm 5.4 \mathrm{~ms}$ $(n=9)\left(\tau_{\text {slow }}\right)$. In contrast, the outward current following a prepulse to $-30 \mathrm{mV}$ comprised only one slow component, as current decay was best fit by a single exponential with a time constant of $235.7 \pm 6.3 \mathrm{~ms}(n=7)$.

The subtraction of the two current traces enabled to isolate A-type-like currents $\left(I_{\text {fast }}\right)$, which were quickly activated ( $\leq 3 \mathrm{~ms}$ ), indicating a strong dependency on voltage for inactivation. The current decay was best fit with a single 


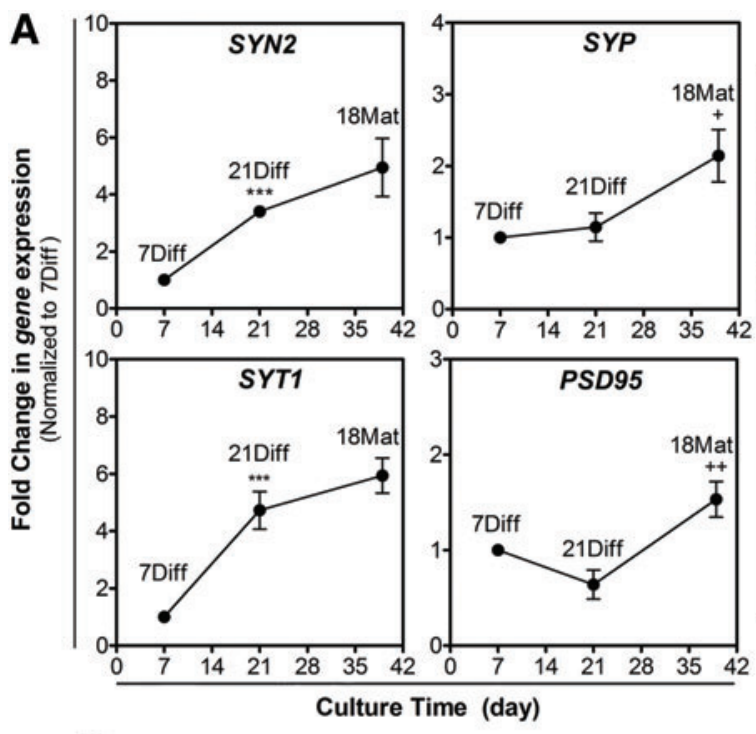

B
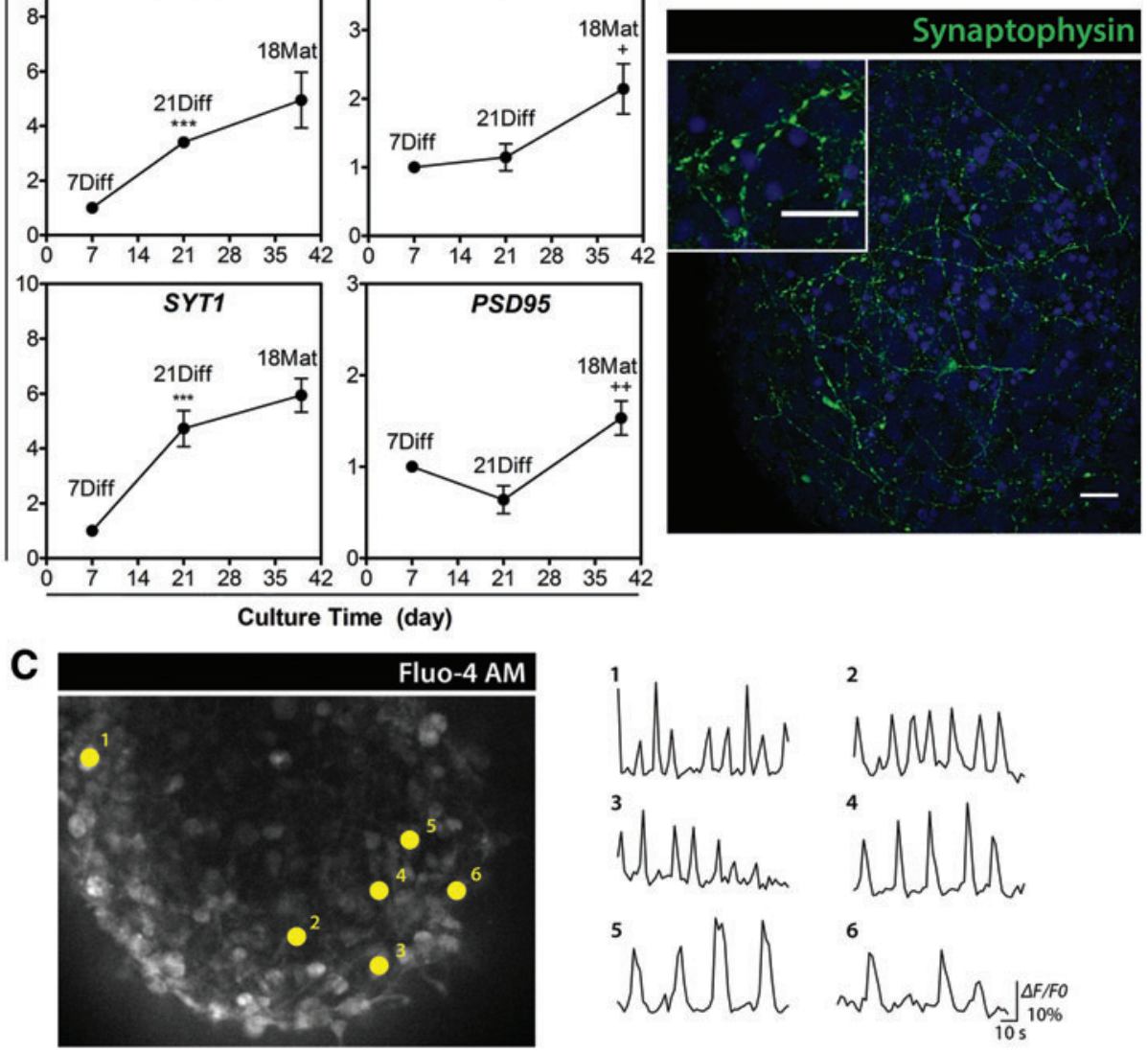

6

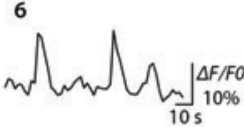

D

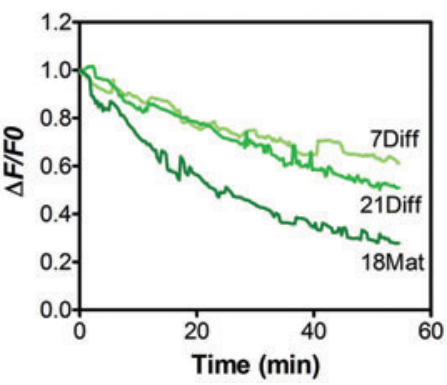

E

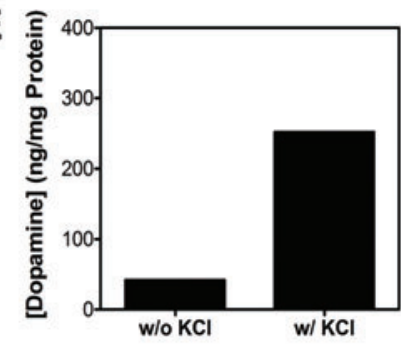

FIG. 7. Synaptic marker enrichment and synaptic vesicle trafficking in hmNPC neurospheres along differentiation. (A) qRT-PCR analysis of $S Y N 2, S Y P, S Y T 1$, and $P S D 95$ gene expression. Fold changes in gene expression were normalized to 7Diff. Data are mean \pm SEM of three independent cultures; asterisks indicate significant difference $(* * * p<0.001$ vs. 7Diff; ${ }^{+} p<0.05,{ }^{++} p<0.01$ vs. 21 Diff) by a one-way ANOVA analysis with a Tukey's post-hoc multiple-comparison test. (B) Confocal immunofluorescence microscopy of whole neurospheres from 18Mat. Maximum-intensity z-projections of 16 optical sections of $0.5 \mu \mathrm{m}$. Detection of synaptophysin; nuclei were labeled with TO-PRO-3. Scale bars $=20 \mu \mathrm{m}$. (C) Spontaneous $\mathrm{Ca}^{2+}$ oscillations in differentiated neurospheres at day 18Mat. Analysis of $\mathrm{Ca}^{2+}$ oscillations in individual neurons (numbers correspond to individual cells analyzed simultaneously); data are from one representative experiment of three independent experiments. (D) Fluorescence intensity analysis of FM 1-43 dye in exocytosis-inducing conditions; data are from one representative experiment of three independent experiments. (E) Dopamine release quantification with and without $\mathrm{KCl}$ stimuli; data are from one representative experiment of three independent experiments. qRT-PCR, quantitative reverse transcription polymerase chain reaction. Color images available online at www.liebertpub.com/tea

exponential with time constant of $23.4 \pm 1.1 \mathrm{~ms} \quad(n=7)$, values of the same range as those reported as typical for Atype current. ${ }^{36} \mathrm{~K}^{+}$currents were also characterized in terms of voltage dependence of activation (Fig. 8B), where current records were converted to conductance, and steady-state inactivation (Fig. 8C). Data were fit with the Boltzmann equation (Eq. 1) obtaining for activation a $V_{1 / 2}$ value of
$-0.3 \pm 0.4 \mathrm{mV}(n=4)$ and $5.3 \pm 0.5 \mathrm{mV}(n=4)$, for $I_{\text {fast }}$ and $I_{\text {slow }}$, respectively. As for inactivation a $V_{1 / 2}$ value of $-81.3 \pm 1.6 \mathrm{mV}(n=3)$ was obtained.

As midbrain mainly receives glutamatergic projections from other surrounding brain regions, ${ }^{37}$ glutamate-evoked ionic currents were evaluated to assess the functionality of glutamate receptors. Glutamate was added to clamped cells 
A

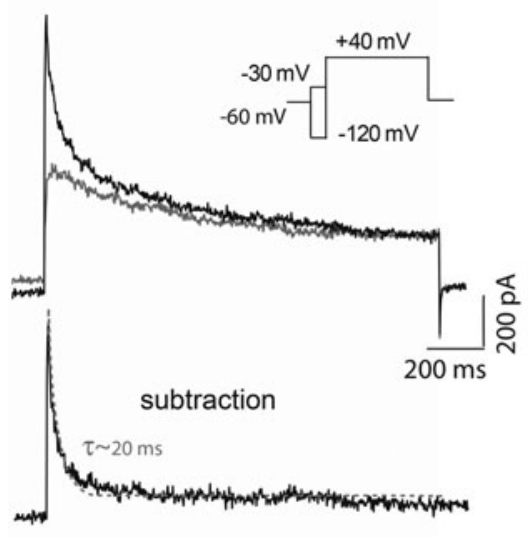

B

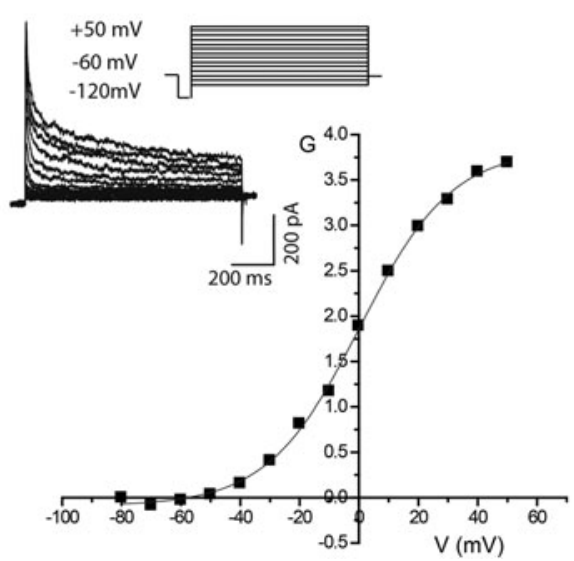

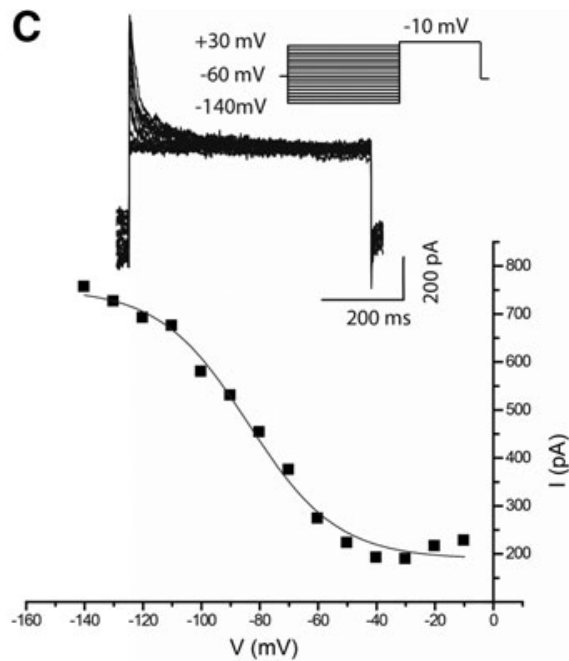

FIG. 8. Voltage-activated potassium $\left(\mathrm{K}_{\mathrm{v}}\right)$ currents in hmNPC-differentiated neurospheres. (A) Whole-cell voltageactivated $\mathrm{K}^{+}$current was evoked by a depolarizing step to $+40 \mathrm{mV}$ for $0.8 \mathrm{~s}$ (holding potential of $-60 \mathrm{mV}$ ) preceded by a prepulse of $150 \mathrm{~ms}$ to $-120 \mathrm{mV}$ (black trace) or to $-30 \mathrm{mV}$ (gray trace). On the bottom the resultant subtraction of both currents is shown to isolate the fast-current component (A-type), which was best fit by a single exponential (discontinuous line) with a time constant of $20.1 \mathrm{~ms}$. (B, C) Voltage dependence of steady-state activation (B) and inactivation (C). (B) Currents evoked by depolarizing steps in increments of $10 \mathrm{mV}$ (1040 ms in duration) following a $-120 \mathrm{mV}$ hyperpolarizing prepulse $(150 \mathrm{~ms}$ in duration from holding potential of $-60 \mathrm{mV})$. Consequent activation curve of peak current $\left(V_{1 / 2}=\right.$ $-0.3 \pm 0.4 \mathrm{mV} ; n=4)$. (C) Currents evoked by a command pulse to $+40 \mathrm{mV}(400 \mathrm{~ms}$ in duration) preceded by prepulses from -140 to $+30 \mathrm{mV}(600 \mathrm{~ms}$ in duration from $-60 \mathrm{mV})$, in $10-\mathrm{mV}$ increments. Consequent inactivation curve of peak current $\left(V_{1 / 2}=-81.3 \pm 1.6 \mathrm{mV} ; n=3\right)$.

under different holding potentials, with increased responses observed at a more hyperpolarized potential $(-70 \mathrm{mV})$ and reversed currents obtained under positive potentials (Fig. 9A). A linear current-voltage relationship was obtained with a reversing potential close to $0 \mathrm{mV}$ (Fig. 9B). The observed glutamate-evoked currents were characterized by a dualphase response, which was more noticeable over a longer period of time (Fig. 9C), with a faster response followed by a slower and more sustained current. In the presence of antagonists of AMPA/kainate and NMDA glutamate receptors (10 $\mu \mathrm{M} \mathrm{CNQX}$ and $10 \mu \mathrm{M} \mathrm{AP}$, respectively), no or small responses to glutamate were registered (Fig. 9D). After 15 min washing, the same clamped cell $(n=3)$ exhibited a robust response to glutamate, confirming the presence and specific activation of glutamate receptors during glutamate application.

\section{Discussion}

In this study, we demonstrated that hmNPCs can be efficiently differentiated within neurospheres into functional dopaminergic and GABAergic neurons and cells from the astrocytic and oligodendrocytic lineages, and be maintained in long-term in vitro cultures. By providing homogeneous aggregation and efficient differentiation of hmNPCs, with establishment of 3D cell-cell interactions, we attained an efficient scalable culture system that is directly transferable to feeding of HTS platforms. These features are critical for basic research on the pathways responsible for the onset and progression of human neurological disorders, as well as for drug discovery.

The cell model presented herein can represent an alternative to organotypic cultures in which cytoarchitecture and cellular functionality of the original tissue $e^{6,38}$ are preserved but fail in maintaining long-term cellular viability and phenotype and present limited availability.

hmNPCs have been initially established and extensively described in 2D culture systems, demonstrating an efficient differentiation into the dopaminergic lineage. ${ }^{11,39,40}$ Nevertheless, these 2D culture systems recapitulate to a lower extent the three-dimensional cell-cell interactions of the brain. We have previously demonstrated that hmNPCs can also be efficiently differentiated as neurospheres, expressing several neuronal and dopaminergic-specific markers after 14 days of differentiation. ${ }^{10}$

In this work, we further explored the neuronal differentiation process, successfully aiding the progression into later developmental stages, attaining maturation and synaptic functionality, by mimicking the developmental stages that multipotent mesencephalic progenitors undergo to acquire a mature dopaminergic neuronal phenotype. Differentiation of hmNPC neurospheres activated the expression of NURRI, with significant upregulation of the mature dopaminergic markers, Pitx3, TH, and DRD2, suggesting the preservation of midbrain region identity and developmental programs. These depend on the integration of various external cues culminating in the activation of signaling pathways, such as the Wnt/ $\beta$-catenin canonical pathway that mediates the proliferation and differentiation of NURR $1^{+}$immature dopaminergic neurons. ${ }^{41,42}$ In later stages of differentiation, NURR1 is required for dopaminergic neuron specification and maturation, regulating the expression of mature markers, such as Pitx3 and TH. ${ }^{43-45}$

Moreover, the expression of vGAT and the ability of differentiated neurospheres to synthesize and release GABA upon stimuli suggested the presence of a GABAergic 

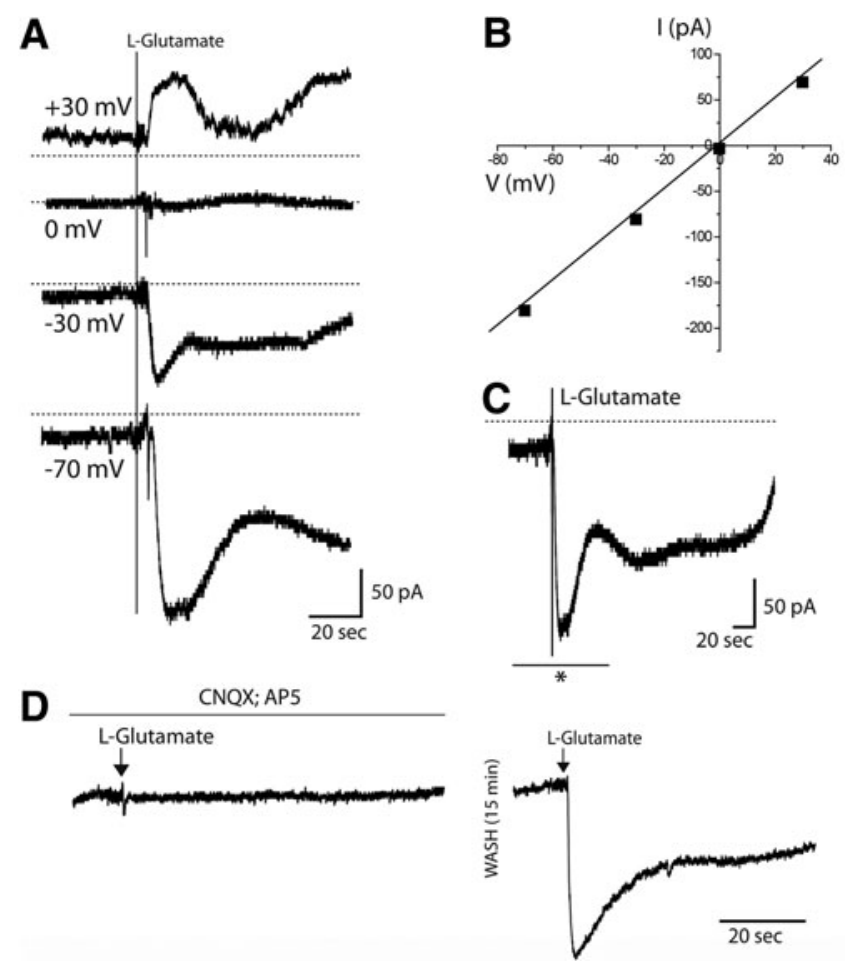

FIG. 9. Whole-cell voltage-clamp recordings of glutamate-gated currents in hmNPC-differentiated neurospheres. (A) Glutamate-gated responses from holding potentials of $+30,0,-30$, and $-70 \mathrm{mV}$. (B) Peak current-voltage $(I-V)$ relationship of glutamate-evoked responses. (C) Glutamateevoked response displayed with longer period to show typical dual response to glutamate [*represents time detail of recording at $-70 \mathrm{mV}$ in the bottom panel of (A)]. (D) Glutamate-gated current inhibition by CNQX and AP5 (AMPA/kainate and NMDA antagonists, respectively), response recovered upon 15-min wash.

population, as previously demonstrated in 2D cultures of differentiated hmNPCs. ${ }^{46}$ These evidences further suggest that the developed 3D model can mimic the main midbrain developmental pathways, generating heterogeneous neurospheres with the two neuronal subpopulations found in the human midbrain, dopaminergic and GABAergic. ${ }^{37}$

Extension of the differentiation process was performed in presence of CAMP, which has been described to promote the differentiation, maturation, and survival of midbrain dopaminergic neurons. ${ }^{47,48}$ Moreover, cAMP has been reported to enhance neuronal differentiation in NPCs derived from other brain regions, such as forebrain in murine NPCs, ${ }^{49}$ and also in other pluri/multipotent cells, such as mesenchymal stem cells, inducing the expression of NURR 1 and $T H^{50}$

The transition from multipotent neural progenitors toward a mixed culture of differentiated neural cells induced modulation of cellular metabolism. The maintenance of a highly glycolytic phenotype despite lower glucose consumption, together with increased pyruvate consumption, suggested the recapitulation of metabolic features of mature neural cells. Both neurons and astrocytes can utilize extracellular pyruvate $^{51}$ and production of lactate from pyruvate has been reported for neurons cultured in glucose-containing medium as a faster way to recycle $\mathrm{NAD}^{+}$produced in glycolysis. ${ }^{52}$ Neural reliance on glycolytic metabolism has been linked to the high energy requirements of the brain, where glycolysis may act as a fast-response pathway to accommodate high ATP demands, namely, to enable the constant activity of $\mathrm{Na}^{+} / \mathrm{K}^{+}$ ATPases, essential for maintenance of neuronal membrane ionic gradients ${ }^{53,54}$ and/or provide local ATP supply to molecular motors. ${ }^{55}$

Differentiated neurospheres presented significantly increased accumulation of pyroglutamate, which can derive from degradation of proteins containing modified N-terminal glutamic acid residues or from glutamate/glutamine cyclization $^{56}$ and has been suggested to act as a reservoir of neural glutamate, the main excitatory neurotransmitter in the $\mathrm{CNS}^{57}$

The accumulation of BCAA catabolism intermediates (2oxoisocaproate and methylsuccinate) points to recapitulation of important astrocyte-neuron nitrogen shuttling systems of human $\mathrm{CNS}^{58}{ }^{5}$ complementing the extensively described glutamine-glutamate cycle between neurons and astrocytes. ${ }^{54}$ Our results suggest that establishment of some of these nitrogen shuttles may occur in an early stage of CNS development and maturation.

The developed cell model, in combination with NMR and/or mass spectrometry analyses of isotopic $\left({ }^{13} \mathrm{C}\right.$ and $\left.{ }^{15} \mathrm{~N}\right)$ tracer studies, can be applied in depicting these neural metabolic shuttles and contribute to increase the mechanistic understanding on the correlation between cell metabolism and stemness/cell identity determination driven by genetic and epigenetic switches. ${ }^{59}$

Upon differentiation, neurospheres exhibited extensive ECM composition remodeling and morphological rearrangements. Gene expression results evidenced a closer resemblance to the in vivo neural ECM, which is mainly comprised of glycosaminoglycans, namely, hyaluronan, heparan sulfate proteoglycans, and chondroitin sulfate proteoglycans and displays low levels of fibrilliar proteins, such as collagens, fibronectin, and laminin, ${ }^{31,60}$ regularly used as matrix in 2D cultures. This was accompanied by changes in plasma membrane architecture, with a transition from highly prevalent lamellipodia found in undifferentiated neurospheres toward a dense filopodia network in differentiated neurospheres. Filopodia have been shown to be paramount in dendritic branching, axonal development, and synapse formation. ${ }^{61}$ Formation of newborn synapses is concomitant with an increase in expression of synaptic markers. ${ }^{62} \mathrm{Ex}-$ pression levels of synaptic proteins, such as SYN2, SYP, SYT1, and PSD95, steadily increased during hmNPC neurosphere differentiation. Moreover, synaptophysinpositive organelles could be detected in puncta along neuronal processes, suggesting that these neurons are fully competent for the biogenesis and clustering of synaptic vesicles.

Neurons in differentiated neurospheres were able to elicit spontaneously $\mathrm{Ca}^{2+}$ oscillations at frequencies reminiscent of action potential firing, as well as to respond to depolarization stimuli leading to FM-1-43 and neurotransmitter release (dopamine and GABA). These results indicate that midbrain neurons generated in hmNPC neurospheres contain functional synapses, where the rapid influx of $\mathrm{Ca}^{2+}$ through voltage-dependent $\mathrm{Ca}^{2+}$ channels arriving in the nerve terminals triggers fusion of neurotransmitter-containing vesicles with the plasma membrane, leading to neurotransmitter 
release into the synaptic shaft. ${ }^{63}$ Further, the electrophysiology recordings, which to our knowledge are the first recordings from single cells within the $3 \mathrm{D}$ structure of neurospheres, demonstrated that the generated neurons were fully polarized $\left(V_{\mathrm{m}}\right.$ of approximately $\left.-70 \mathrm{mV}\right)$ at potentials modulated by functional voltage-activated ion channels. The registered voltage-activated $\mathrm{K}^{+}$currents pointed out for the existence of at least two populations of voltage-gate $\mathrm{K}^{+}$ channels $\left(K_{\mathrm{V}}\right)$, one underlying $I_{\text {fast }}$ and other underlying $I_{\text {slow }}$. The conspicuous $I_{\text {fast }}$ component showed typical patterns of an A-type current, which are perceived to have high relevance in numerous physiological and pathological contexts. From the $K_{\mathrm{V}}$ channels/subunits that are known to be responsible to trigger A-type currents, two emerge as probable candidates to underlie the $I_{\text {fast }}$ component. Considering that this current showed (1) a voltage profile for activation with a $V_{1 / 2}$ of around $-10 \mathrm{mV}$ (considering a $-9 \mathrm{mV}$ junctional potential), which points out for high threshold activating channels; (2) a hyperpolarized voltage dependence of inactivation; and, most noticeably, (3) a time constant for the inactivation time course in the order of $20 \mathrm{~ms}$, the channels $K_{\mathrm{V}} 1.4$ and $K_{\mathrm{V}} 4.2$ are envisaged to be the most likely candidates to be present in neurons of hmNPC-differentiated neurospheres. Indeed, extensive characterization on a predominant A-type current in hmNPCs differentiated in 2D cultures was reported, ${ }^{64}$ which according to the authors is evoked by $K_{\mathrm{V}} 4.2$ channels. Nevertheless, one cannot exclude the possibility of $K_{\mathrm{V}} 1.4$ channels to be also present in the differentiated neurospheres.

Moreover, the excitability of differentiated hmNPC neurospheres was challenged by the addition of glutamate, with the generated neurons demonstrating to be able to elicit glutamate-gated currents with two clear components, one faster followed by a slower and more sustained current. The linear voltage-current relationship reversing close to $0 \mathrm{mV}$ corroborates the involvement of channels with unspecific cationic conductance. Further, these currents were blocked by coincubation of AMPA/kainate and NMDA antagonists (CNQX and AP5, respectively). Altogether, these results showed that hmNPC neurospheres contain mature neurons with functional glutamate receptors, which are most likely AMPA/kainate and NMDA receptor types, as well as the associated channels. These observations are in agreement to what was described previously for hmNPC differentiated in 2D cultures. ${ }^{65}$ The presence of functional postsynaptic glutamatergic molecular machinery further suggests that the developed 3D model recapitulates in vivo features, since the midbrain is known to receive and integrate multiple glutamatergic inputs from other brain regions. $^{37}$

In this work, we established a novel culture system that yields a reproducible human 3D CNS cell model enriched in dopaminergic neurons. By combining scalable protocols with an extensive toolbox of characterization methods, we have generated a comprehensive set of developmental data on the in vitro differentiation of human midbrain neurons. This approach can be extended to other sources of human neural stem cells, such as patient-derived iPSCs, based on recent reports on the generation of regionally specified neural progenitors from human pluripotent stem cells under defined conditions. ${ }^{66}$ The exploitation of these novel cellular models is likely to boost our mechanistic understanding of the pathogenesis of human disorders as well as accelerate the discovery of new therapeutics.

\section{Acknowledgments}

The authors gratefully acknowledge Dr. Johannes Schwarz for the supply of hmNPCs within the scope of the EU project BrainCAV (FP7-222992), Pedro Almada and Dr. Emilio Gualda for support on confocal microscopy, Dr. Ana Amaral for support on hmNPC 3D cultures and, Dr. Rodolfo Negri and Dr. Enrico Tagliafico for chip bioinformatics. IBET Analytical Services Unit, Portugal, is acknowledged for HPLC analysis and Institute of Ophthalmology, UCL, United Kingdom, is acknowledged for collection of SBFSEM data. The NMR spectrometers are part of The National NMR Facility, supported by Fundação para a Ciência e a Tecnologia (RECI/ BBB-BQB/0230/2012). This work was supported by BrainCAV (FP7-222992) and Brainvectors (FP7-286071); funded by the EU (PTDC/EBB-BIO/112786/2009 and PTDC/EBBBIO/119243/2010); funded by Fundação para a Ciência e Tecnologia, Portugal; and Cancer Research, United Kingdom. D.S. was recipient of a $\mathrm{PhD}$ fellowship from FCT, Portugal (SFRH/BD/78308/2011, FCT).

\section{Disclosure Statement}

No competing financial interests exist.

\section{References}

1. Lin, R.-Z., Lin, R.-Z., and Chang, H.-Y. Recent advances in three-dimensional multicellular spheroid culture for biomedical research. Biotechnol J 3, 1172, 2008.

2. Griffith, L.G., and Swartz, M.A. Capturing complex 3D tissue physiology in vitro. Nat Rev Mol Cell Biol 7, 211, 2006.

3. Miller, G. Is pharma running out of brainy ideas? Science 329, 502, 2010.

4. Schüle, B., Pera, R.A.R., and Langston, J.W. Can cellular models revolutionize drug discovery in Parkinson's disease? Biochim Biophys Acta 1792, 1043, 2009.

5. Fennema, E., Rivron, N., Rouwkema, J., van Blitterswijk, C., and de Boer, J. Spheroid culture as a tool for creating 3D complex tissues. Trends Biotechnol 31, 108, 2013.

6. Pampaloni, F., Reynaud, E.G., and Stelzer, E.H.K. The third dimension bridges the gap between cell culture and live tissue. Nat Rev Mol Cell Biol 8, 839, 2007.

7. Potter, W., Kalil, R.E., and Kao, W.J. Biomimetic material systems for neural progenitor cell-based therapy. Front Biosci 13, 806, 2008.

8. Breslin, S., and O'Driscoll, L. Three-dimensional cell culture: the missing link in drug discovery. Drug Discov Today 18, 240, 2013.

9. Moors, M., Rockel, T.D., Abel, J., Cline, J.E., Gassmann, K., Schreiber, T., et al. Human neurospheres as threedimensional cellular systems for developmental neurotoxicity testing. Environ Health Perspect 117, 1131, 2009.

10. Brito, C., Simão, D., Costa, I., Malpique, R., Pereira, C.I., Fernandes, P., et al. 3D cultures of human neural progenitor cells: dopaminergic differentiation and genetic modification. Methods 56, 452, 2012.

11. Storch, A., Paul, G., Csete, M., Boehm, B.O., Carvey, P.M., Kupsch, A., et al. Long-term proliferation and dopaminergic differentiation of human mesencephalic neural precursor cells. Exp Neurol 170, 317, 2001. 
12. Wegner, F., Kraft, R., Busse, K., Härtig, W., Ahrens, J., Leffler, A., et al. Differentiated human midbrain-derived neural progenitor cells express excitatory strychninesensitive glycine receptors containing $\alpha 2 \beta$ subunits. PLoS One 7, e36946, 2012.

13. Serra, M., Correia, C., Malpique, R., Brito, C., Jensen, J., Bjorquist, P., et al. Microencapsulation technology: a powerful tool for integrating expansion and cryopreservation of human embryonic stem cells. PLoS One 6, e23212, 2011.

14. Deerinck, T.J., Bushong, E.A., Thor, A., and Ellisman, M.H. NCMIR methods for 3D EM: a new protocol for preparation of biological specimens for serial block face scanning electron microscopy. Available from: http://ncmir .ucsd.edu/sbfsem-protocol.pdf, 2010 (Last accessed January 19, 2014).

15. Duarte, T.M., Carinhas, N., Silva, A.C., Alves, P.M., and Teixeira, A.P. ${ }^{1}$ H-NMR protocol for exometabolome analysis of cultured mammalian cells. Methods Mol Biol 1104, 237, 2014.

16. Livak, K.J., and Schmittgen, T.D. Analysis of relative gene expression data using real-time quantitative PCR and the 2(-delta delta C(T)) method. Methods 25, 402, 2001.

17. Smyth, G.K. Limma: linear models for microarray data. In: Gentleman, R., Carey, V., Huber, W., Irizarry, R., and Dudoit, S., eds. Bioinformatics and Computational Biology Solutions Using R and Bioconductor. New York: Springer, 2005, pp. 397-420.

18. Maere, S., Heymans, K., and Kuiper, M. BiNGO: a cytoscape plugin to assess overrepresentation of gene ontology categories in biological networks. Bioinformatics 21, 3448, 2005.

19. Benjamini, Y., and Hochberg, Y. Controlling the false discovery rate: a practical and powerful approach to multiple testing. J R Stat Soc Ser B 57, 289, 1995.

20. Lakshmana, M.K., and Raju, T.R. An isocratic assay for norepinephrine, dopamine, and 5-hydroxytryptamine using their native fluorescence by high-performance liquid chromatography with fluorescence detection in discrete brain areas of rat. Anal Biochem 246, 166, 1997.

21. Carinhas, N., Bernal, V., Monteiro, F., Carrondo, M.J.T., Oliveira, R., and Alves, P.M. Improving baculovirus production at high cell density through manipulation of energy metabolism. Metab Eng 12, 39, 2010.

22. Lima, P.A., and Marrion, N.V. Mechanisms underlying activation of the slow AHP in rat hippocampal neurons. Brain Res 1150, 74, 2007.

23. Vicente, M.I., Costa, P.F., and Lima, P.A. Galantamine inhibits slowly inactivating $\mathrm{K}+$ currents with a dual doseresponse relationship in differentiated N1E-115 cells and in CA1 neurones. Eur J Pharmacol 634, 16, 2010.

24. Nishio, T., Furukawa, S., Akiguchi, I., and Sunohara, N. Medial nigral dopamine neurons have rich neurotrophin support in humans. Neuroreport 9, 2847, 1998.

25. Numan, S., Gall, C.M., and Seroogy, K.B. Developmental expression of neurotrophins and their receptors in postnatal rat ventral midbrain. J Mol Neurosci 27, 245, 2005.

26. Zaman, V., Nelson, M.E., Gerhardt, G.A., and Rohrer, B. Neurodegenerative alterations in the nigrostriatal system of trkB hypomorphic mice. Exp Neurol 190, 337, 2004.

27. Jontes, J.D., and Smith, S.J. Filopodia, spines, and the generation of synaptic diversity. Neuron 27, 11, 2000.

28. Lendvai, B., Stern, E.A., Chen, B., and Svoboda, K. Experience-dependent plasticity of dendritic spines in the developing rat barrel cortex in vivo. Nature 404, 876, 2000.
29. Iwama, E., Tsuchimoto, D., Iyama, T., Sakumi, K., Nakagawara, A., Takayama, K., et al. Cancer-related PRUNE2 protein is associated with nucleotides and is highly expressed in mature nerve tissues. J Mol Neurosci 44, 103, 2011.

30. Bandtlow, C.E., and Zimmermann, D.R. Proteoglycans in the developing brain: new conceptual insights for old proteins. Physiol Rev 80, 1267, 2000.

31. Dwyer, C., and Matthews, R. The neural extracellular matrix, cell adhesion molecules and proteolysis in glioma invasion and tumorigenicity. In: Garami, M., ed. Molecular Targets of CNS Tumors. Rijeka: InTech, 2011, pp. 239-264.

32. Gaffield, M.A., and Betz, W.J. Imaging synaptic vesicle exocytosis and endocytosis with FM dyes. Nat Protoc 1, 2916, 2006.

33. Hoopmann, P., Rizzoli, S.O., and Betz, W.J. Imaging synaptic vesicle recycling by staining and destaining vesicles with FM dyes. Cold Spring Harb Protoc 2012, 77, 2012.

34. Ikegaya, Y., Le Bon-Jego, M., and Yuste, R. Large-scale imaging of cortical network activity with calcium indicators. Neurosci Res 52, 132, 2005.

35. Tashiro, A., Goldberg, J., and Yuste, R. Calcium oscillations in neocortical astrocytes under epileptiform conditions. J Neurobiol 50, 45, 2002.

36. Coetzee, W.A., Amarillo, Y., Chiu, J., Chow, A., Lau, D., McCormack, T., et al. Molecular diversity of $\mathrm{K}+$ channels. Ann N Y Acad Sci 868, 233, 1999.

37. Korotkova, T.M., Ponomarenko, A.A., Brown, R.E., and Haas, H.L. Functional diversity of ventral midbrain dopamine and GABAergic neurons. Mol Neurobiol 29, 243, 2004.

38. Bodea, G.O., and Blaess, S. Organotypic slice cultures of embryonic ventral midbrain: a system to study dopaminergic neuronal development in vitro. J Vis $\operatorname{Exp}(59)$, e3350, 2012.

39. Schwarz, J. Developmental perspectives on human midbrain-derived neural stem cells. Parkinsonism Relat Disord 13(S3), S466, 2007.

40. Schaarschmidt, G., Schewtschik, S., Kraft, R., Wegner, F., Eilers, J., Schwarz, J., et al. A new culturing strategy improves functional neuronal development of human neural progenitor cells. J Neurochem 109, 238, 2009.

41. Joksimovic, M., Yun, B.A., Kittappa, R., Anderegg, A.M., Chang, W.W., Taketo, M.M., et al. Wnt antagonism of Shh facilitates midbrain floor plate neurogenesis. Nat Neurosci 12, 125, 2009.

42. Alves dos Santos, M.T.M., and Smidt, M.P. En1 and Wnt signaling in midbrain dopaminergic neuronal development. Neural Dev 6, 1, 2011.

43. Ang, S.-L. Transcriptional control of midbrain dopaminergic neuron development. Development 133, 3499, 2006.

44. Prakash, N., and Wurst, W. Genetic networks controlling the development of midbrain dopaminergic neurons. J Physiol 575, 403, 2006.

45. Abeliovich, A., and Hammond, R. Midbrain dopamine neuron differentiation: factors and fates. Dev Biol 304, 447, 2007.

46. Wegner, F., Kraft, R., Busse, K., Härtig, W., Schaarschmidt, G., Schwarz, S.C., et al. Functional and molecular analysis of GABA receptors in human midbrain-derived neural progenitor cells. J Neurochem 107, 1056, 2008.

47. Goldberg, J.L., and Barres, B.A. The relationship between neuronal survival and regeneration. Annu Rev Neurosci 23, 579, 2000.

48. Michel, P.P., and Agid, Y. Chronic activation of the cyclic AMP signaling pathway promotes development and long-term 
survival of mesencephalic dopaminergic neurons. J Neurochem 67, 1633, 1996.

49. Zahir, T., Chen, Y.F., MacDonald, J.F., Leipzig, N., Tator, C.H., and Shoichet, M.S. Neural stem/progenitor cells differentiate in vitro to neurons by the combined action of dibutyryl cAMP and interferon-gamma. Stem Cells Dev 18, 1423, 2009.

50. Tio, M., Tan, K.H., Lee, W., Wang, T.T., and Udolph, G. Roles of db-cAMP, IBMX and RA in aspects of neural differentiation of cord blood derived mesenchymal-like stem cells. PLoS One 5, e9398, 2010.

51. Zwingmann, C., and Leibfritz, D. Regulation of glial metabolism studied by 13C-NMR. NMR Biomed 16, 370, 2003.

52. Cruz, F., Villalba, M., García-Espinosa, M.A., Ballesteros, P., Bogónez, E., Satrústegui, J., et al. Intracellular compartmentation of pyruvate in primary cultures of cortical neurons as detected by (13)C NMR spectroscopy with multiple (13)C labels. J Neurosci Res 66, 771, 2001.

53. Fornazari, M., Nascimento, I.C., Nery, A.A., da Silva, C.C.C., Kowaltowski, A.J., and Ulrich, H. Neuronal differentiation involves a shift from glucose oxidation to fermentation. J Bioenerg Biomembr 43, 531, 2011.

54. Magistretti, P.J., and Allaman, I. Brain energy metabolism. In: Pfaff, D.W., ed. Neuroscience in the 21st Century: From Basic to Clin. New York: Springer, 2013, pp. 1591-1620.

55. Zala, D., Hinckelmann, M.-V., Yu, H., Lyra da Cunha, M.M., Liot, G., Cordelières, F.P., et al. Vesicular glycolysis provides on-board energy for fast axonal transport. Cell 152, 479, 2013.

56. Schilling, S., Wasternack, C., and Demuth, H.-U. Glutaminyl cyclases from animals and plants: a case of functionally convergent protein evolution. Biol Chem 389, 983, 2008.

57. Kumar, A., and Bachhawat, A.K. Pyroglutamic acid: throwing light on a lightly studied metabolite. Curr Sci 102, 288, 2012.

58. Bixel, M.G., Engelmann, J., Willker, W., Hamprecht, B., and Leibfritz, D. Metabolism of [U-(13)C]leucine in cultured astroglial cells. Neurochem Res 29, 2057, 2004.

59. Folmes, C.D.L., Nelson, T.J., Dzeja, P.P., and Terzic, A. Energy metabolism plasticity enables stemness programs. Ann N Y Acad Sci 1254, 82, 2012.
60. Ruoslahti, E. Brain extracellular matrix. Glycobiology 6, 489, 1996.

61. Zito, K., Knott, G., Shepherd, G.M.G., Shenolikar, S., and Svoboda, K. Induction of spine growth and synapse formation by regulation of the spine actin cytoskeleton. Neuron 44, 321, 2004.

62. Valtorta, F., Pozzi, D., Benfenati, F., and Fornasiero, E.F. The synapsins: multitask modulators of neuronal development. Semin Cell Dev Biol 22, 378, 2011.

63. Evans, G.J.O., and Cousin, M.A. Simultaneous monitoring of three key neuronal functions in primary neuronal cultures. J Neurosci Methods 160, 197, 2007.

64. Schaarschmidt, G., Wegner, F., Schwarz, S.C., Schmidt, H., and Schwarz, J. Characterization of voltage-gated potassium channels in human neural progenitor cells. PLoS One 4, e6168, 2009.

65. Wegner, F., Kraft, R., Busse, K., Schaarschmidt, G., Härtig, W., Schwarz, S.C., et al. Glutamate receptor properties of human mesencephalic neural progenitor cells: NMDA enhances dopaminergic neurogenesis in vitro. J Neurochem 111, 204, 2009.

66. Kirkeby, A., Grealish, S., Wolf, D.A., Nelander, J., Wood, J., Lundblad, M., et al. Generation of regionally specified neural progenitors and functional neurons from human embryonic stem cells under defined conditions. Cell Rep 1, 703,2012
Address correspondence to: Catarina Brito, PhD iBET-Instituto de Biologia Experimental e Tecnológica Apartado 12 Oeiras 2780-901 Portugal

E-mail: anabrito@itqb.unl.pt

Received: January 28, 2014 Accepted: September 15, 2014 Online Publication Date: November 6, 2014 